\title{
Discrépance et diaphonie en dimension un
}

\author{
par \\ Henri Chaix et Henri Faure (Marseille)
}

1. Introduction. Cette étude est consacrée essentiellement à la discrépance quadratique $T$ et à la diaphonie $F$ des suites de van der Corput généralisées (voir les paragraphes 2 et 3 pour les définitions); elle est parallèle à celle, menée une dizaine d'années plus tôt par l'un des auteurs, sur la discrépance extrême et publiée au Bulletin de la S.M.F. [6]; elle est réalisée selon le même schéma : détermination de formules exactes sous forme de séries pour les mesures d'irrégularités de distribution étudiées, puis comportement asymptotique précis de ces mesures, avec prospection poussée à la machine; elle couvre la totalité des travaux effectués précédemment sur le sujet ([1], [12] à [16]). Les résultats détaillés font l'objet du paragraphe 4; nous en donnons ici un aperçu en les situant dans leur contexte historique, en relation avec l'autre famille de suites à faibles irrégularités : les suites $(n \alpha)$. Notons que les paragraphes 2.2, 3.2 et 3.3 ne sont pas nécessaires pour aborder le paragraphe 4 .

Etant donnée une suite infinie $X$ à valeurs dans [0,1], posons

$$
\begin{aligned}
t_{1}(X) & =\limsup _{N \rightarrow+\infty}(T(N, X) / \log N), \\
t_{2}(X) & =\limsup _{N \rightarrow+\infty}\left(T^{2}(N, X) / \log N\right) \text { et } \\
f(X) & =\limsup _{N \rightarrow+\infty}\left(F^{2}(N, X) / \log N\right) .
\end{aligned}
$$

Définissons alors les constantes

$$
\mu_{1}=\inf _{X} t_{1}(X), \quad \mu_{2}=\inf _{X} t_{2}(X) \quad \text { et } \quad \nu=\inf _{X} f(X),
$$

où $X$ parcourt l'ensemble des suites infinies de $[0,1]$. On sait déjà grâce au théorème de Roth [7] que $\mu_{2}>0.00002$, et Proinov [12] a annoncé en 1986 que $\nu>0.0002$.

Pour les suites $(n \alpha)$, Proinov [11] a le premier montré que $\mu_{2}$ et $\nu$ sont finis : $\alpha$ étant le nombre d'or, il obtient

$$
\nu<90.32 \text { et, en symétrisant la suite, } \mu_{2}<9.16 \text {. }
$$


Ces résultats ont récemment été améliorés par Xiao [17] :

$$
\nu<7.5 \text { et } \mu_{2}<0.76 \text {, }
$$

toujours avec $(n \alpha)$ où $\alpha$ est le nombre d'or. Remarquons que $\mu_{1}$ est nulle, puisque $\mu_{2}$ est fini.

Pour les suites de van der Corput, on peut distinguer trois familles imbriquées : la famille originelle des suites $S_{b}^{I}$ dites de van der Corput-Halton, celle des suites $S_{b}^{\sigma}$ où agit une permutation $\sigma$ sur les chiffres de la base $b$ et enfin celle des suites $S_{B}^{\Sigma}$ où la base $B$ est variable et $\Sigma$ est une suite de permutations associée à $B$ (voir le paragraphe 3 ).

En étudiant la famille des suites $S_{b}^{I}$, Proinov et Grozdanov ([15], [16]) ont trouvé des majorations pour $t_{2}\left(S_{b}^{I}\right)$ et $f\left(S_{b}^{I}\right)$, les meilleures de ces majorations obtenues pour $b=2$ conduisent à

$$
\nu<14.24 \text { et } \mu_{2}<1.45 \text { (grâce à la suite symétrisée pour } \mu_{2} \text { ) . }
$$

Toujours dans la même famille et pour $b=2$, une amélioration a été donnée par Proinov et Atanassov [14], avec $\nu<1.59$.

Notre bonne maîtrise des mesures d'irrégularités de distribution des suites $S_{b}^{\sigma}$ nous permet d'obtenir les meilleurs résultats actuels : nous avons des formules exactes pour $t_{1}\left(S_{b}^{\sigma}\right)$ et $f\left(S_{b}^{\sigma}\right)$ qui fournissent d'une part, les majorations

$$
\nu<1.316 \quad \text { et } \quad \mu_{2}<0.134
$$

(avec la base $b=19$ et une permutation bien choisie) et, d'autre part, une autre preuve que $\mu_{1}=0\left(\right.$ avec $\lim _{b \rightarrow+\infty} t_{1}\left(S_{b}^{\sigma_{b}}\right)=0$, où $\sigma_{b}$ est optimale pour $\left.t_{1}\right)$.

En outre, nous sommes en mesure d'effectuer le calcul explicite de $f\left(S_{b}^{I}\right)$ et $t_{1}\left(S_{b}^{I}\right)$.

Enfin, s'agissant de la famille des suites $S_{B}^{\Sigma}$, nous avons des formules exactes sous forme de séries pour $T^{2}$ et $F^{2}$; nous retrouvons ainsi des résultats obtenus par Proinov et Atanassov ([1], [13], [14]) que nous complétons par la construction de familles de contre-exemples relatifs à des conditions suffisantes pour que $T\left(N, S_{B}^{\Sigma}\right)$ et $F^{2}\left(N, S_{B}^{\Sigma}\right)$ soient de l'ordre de $\log N$.

Les paragraphes 5,6 , et 7 sont consacrés respectivement aux démonstrations des formules exactes, des estimations asymptotiques et enfin à l'étude numérique de cas particuliers.

La plupart des résultats ont été annoncés dans les trois notes aux Comptes Rendus de l'Académie des Sciences ([3]-[5]).

\section{Mesures d'irrégularités de distribution}

2.1. Définitions. Nous donnons ici les principales définitions concernant la discrépance pour une suite quelconque. 
Soient $\alpha$ et $\beta$ deux réels de $[0,1]$; on appelle intervalle $[\alpha, \beta[$ l'intervalle semi-ouvert usuel si $\alpha<\beta$, l'ensemble $[0, \beta[\cup[\alpha, 1]$ si $\alpha>\beta$ et l'ensemble vide si $\alpha=\beta$.

DÉFInition 2.1. Etant donnés une suite infinie $X=\left(x_{n}\right)$ à valeurs dans $[0,1]$, un entier $N \geq 1$ et un intervalle $[\alpha, \beta[$, l'écart (ou reste à l'équirépartition) pour $[\alpha, \beta[$ est

$$
E([\alpha, \beta[; N ; X)=A([\alpha, \beta[; N ; X)-N l([\alpha, \beta[),
$$

où $A\left(\left[\alpha, \beta[; N ; X)\right.\right.$ est le nombre d'indices $n$ tels que $1 \leq n \leq N$ et $x_{n} \in$ $[\alpha, \beta[$ et où $l([\alpha, \beta[)$ est la longueur de $[\alpha, \beta[$.

Pour simplifier, nous noterons $E(\alpha, N, X)=E([0, \alpha[; N ; X)$.

Remarquons que

$$
E([\alpha, \beta[; N ; X)=-E([\beta, \alpha[; N ; X)=E(\beta, N, X)-E(\alpha, N, X) .
$$

DÉfinition 2.2. La discrépance extrême à l'origine de la suite $X$ au rang $N$ est définie par

$$
D^{*}(N, X)=\sup _{0 \leq \alpha \leq 1}|E(\alpha, N, X)|,
$$

et la discrépance extrême par

$$
D(N, X)=\sup _{0 \leq \alpha<\beta \leq 1} \mid E([\alpha, \beta[; N ; X) \mid .
$$

DÉfinition 2.3. Pour un réel $p \geq 1$, la discrépance $L^{p}$ de la suite $X$ au rang $N$ est définie par

$$
D^{(p)}(N, X)=\left(\int_{0}^{1}|E(\alpha, N, X)|^{p} d \alpha\right)^{1 / p} .
$$

R e m arque. La discrépance quadratique $D^{(2)}$ est souvent désignée par $T$, et la discrépance extrême à l'origine $D^{*}$ aussi par $D^{(\infty)}$.

Une autre notion, introduite par Zinterhof [19] en 1976, est la diaphonie.

DÉfinition 2.4. La diaphonie de la suite $X$ au rang $N$ est définie par

$$
F(N, X)=\left(2 \sum_{m=1}^{\infty}\left|\sum_{n=1}^{N} \exp \left(2 i \pi m x_{n}\right)\right|^{2} / m^{2}\right)^{1 / 2} .
$$

Remarque. Certains auteurs ([9], [11]) divisent par $N$ ces formules de définition.

2.2. Propriétés. Nous donnons ci-dessous des propriétés que nous utiliserons par la suite. 
Propriété 2.1. On a les relations

$$
D^{(p)} \leq D^{(q)} \leq D^{*} \leq D \leq 2 D^{*}
$$

où les réels $p$ et $q$ vérifient $1 \leq p<q$.

Propriété 2.2 (Formule de Koksma; [9], p. 110). Pour toute suite $X$ et tout entier $N \geq 1$, on a

$$
T^{2}(N, X)=\left(\sum_{n=1}^{N}\left(1 / 2-x_{n}\right)\right)^{2}+F^{2}(N, X) /\left(4 \pi^{2}\right) .
$$

PropriÉté 2.3. Pour toute suite $X$ et tout entier $N \geq 1$, on a

$$
F^{2}(N, X)=2 \pi^{2} \int_{0}^{1} \int_{0}^{1} E^{2}([\alpha, \beta[; N ; X) d \alpha d \beta .
$$

Preuve. La démonstration résulte du fait que

$$
\begin{aligned}
\int_{0}^{1} \int_{0}^{1} E^{2}([\alpha, \beta[; N ; X) d \alpha d \beta & =\int_{0}^{1} \int_{0}^{1}(E(\beta, N, X)-E(\alpha, N, X))^{2} d \alpha d \beta \\
& =2 \int_{0}^{1} E^{2}(\alpha, N, X) d \alpha-2\left(\int_{0}^{1} E(\alpha, N, X) d \alpha\right)^{2},
\end{aligned}
$$

que

$$
\int_{0}^{1} E(\alpha, N, X) d \alpha=\sum_{n=1}^{N}\left(1 / 2-x_{n}\right)
$$

et de la formule de Koksma (propriété 2.2).

Propriété 2.4. Soit une suite $X$ à valeurs dans $[0,1]$; si à tout réel $t$ de $\left[0,1\left[\right.\right.$, on associe la suite $X^{\prime}$ définie par

alors

$$
x_{n}^{\prime}=x_{n}+t(\bmod 1),
$$

$$
D\left(N, X^{\prime}\right)=D(N, X) \quad \text { et } \quad F\left(N, X^{\prime}\right)=F(N, X) .
$$

Preuve. Pour $\alpha$ et $\beta$ réels de [0,1], posons

$$
\alpha^{\prime}=\alpha+t(\bmod 1) \quad \text { et } \quad \beta^{\prime}=\beta+t(\bmod 1) \text {; }
$$

on vérifie facilement que $A\left(\left[\alpha, \beta[; N ; X)=A\left(\left[\alpha^{\prime}, \beta^{\prime}\left[; N ; X^{\prime}\right)\right.\right.\right.\right.$, d'où

$$
E\left(\left[\alpha, \beta[; N ; X)=E\left(\left[\alpha^{\prime}, \beta^{\prime}\left[; N ; X^{\prime}\right) .\right.\right.\right.\right.
$$

Pour $D$, on en déduit la propriété annoncée, en remarquant que

$$
D\left(N, X^{\prime}\right)=\sup _{0 \leq \alpha^{\prime}<\beta^{\prime}<1} \mid E\left(\left[\alpha^{\prime}, \beta^{\prime}\left[; N ; X^{\prime}\right) \mid\right.\right.
$$

car 1 n'est pas terme de $X^{\prime}$. Pour $F$, il suffit de se reporter à la définition 2.4 . 


\section{Suites de van der Corput généralisées et fonctions associées}

\subsection{Définitions}

DÉfinition 3.1 (Suites en base $b$ ). Soient un entier $b \geq 2$ et $\Sigma=\left(\sigma_{j}\right)_{j \geq 0}$ une suite de permutations de $\{0,1, \ldots, b-1\}$; la suite de van der Corput en base b associée à $\Sigma$, notée $S_{b}^{\Sigma}$, est définie [6] par

$$
S_{b}^{\Sigma}(n)=\sum_{j=0}^{\infty} \sigma_{j}\left(a_{j}(n)\right) b^{-j-1}
$$

où $a_{j}(n)$ est la jième décimale de $n-1$ développé en base $b$ :

$$
n-1=\sum_{j=0}^{\infty} a_{j}(n) b^{j}, \quad \text { avec } n \geq 1 \text { et } 0 \leq a_{j}(n)<b .
$$

Rem arque. La suite originelle de van der Corput s'obtient pour $b=2$ et $\Sigma=I$, suite constante égale à la permutation identique; certains auteurs appellent suites de van der Corput-Halton les suites $S_{b}^{I}$.

De la même façon, nous noterons $S_{b}^{\sigma}$ la suite associée à la suite constante $\Sigma=(\sigma)$.

DÉfinition 3.2 (Suites en base variable). Soit $B=\left(b_{j}\right)_{j \geq 0}$, avec $b_{0}=1$ et $b_{j} \geq 2$ pour $j \geq 1$; la suite de van der Corput en base $B$ associée à $\Sigma$ est définie [6] par

$$
S_{B}^{\Sigma}(n)=\sum_{j=0}^{\infty} \sigma_{j}\left(a_{j}(n)\right) / B_{j+1}
$$

où $\Sigma=\left(\sigma_{j}\right)_{j \geq 0}$ avec $\sigma_{j}$ une permutation de $\left\{0,1, \ldots, b_{j+1}-1\right\}, B_{j}=$ $\prod_{i=0}^{j} b_{i}$ et $n-1=\sum_{j=0}^{\infty} a_{j}(n) B_{j}$ (développement en base variable $B$ de $n-1)$.

DÉfinition 3.3 (Fonction $\varphi_{b, h}^{\sigma}$ ). Soit $\mathcal{Z}_{b}^{\sigma}=(\sigma(0) / b, \sigma(1) / b, \ldots$, $\sigma(b-1) / b)$; pour tout entier $h$ tel que $0 \leq h \leq b-1$, la fonction $\varphi_{b, h}^{\sigma}$ est définie, sur $[(k-1) / b, k / b[$, par

$$
\varphi_{b, h}^{\sigma}(x)= \begin{cases}A\left(\left[0, h / b\left[; k ; \mathcal{Z}_{b}^{\sigma}\right)-h x\right.\right. & \text { si } 0 \leq h \leq \sigma(k-1), \\ (b-h) x-A\left(\left[h / b, 1\left[; k ; \mathcal{Z}_{b}^{\sigma}\right)\right.\right. & \text { si } \sigma(k-1)<h<b\end{cases}
$$

où $k$ est un entier tel que $1 \leq k \leq b$. La fonction $\varphi_{b, h}^{\sigma}$, ainsi définie par morceaux sur $[0,1[$, est étendue sur $\mathbb{R}$ par périodicité.

Remarque. Au couple $(b, \sigma)$ sont donc associées $b$ fonctions $\varphi_{b, 0}^{\sigma}=$ $0, \varphi_{b, 1}^{\sigma}, \ldots, \varphi_{b, b-1}^{\sigma}$ qui sont les clés pour exprimer l'écart $E\left(\alpha, N, S_{B}^{\Sigma}\right)$, d'où résulteront les formules pour les discrépances et la diaphonie. Pour cela, il est commode d'introduire les nouvelles fonctions suivantes. 
DÉFINITION 3.4 (Fonctions $\varphi, \phi, \chi, \psi$ ).

$$
\begin{gathered}
\varphi_{b}^{\sigma}=\sum_{h=0}^{b-1} \varphi_{b, h}^{\sigma}, \quad \phi_{b}^{\sigma}=\sum_{h=0}^{b-1}\left(\varphi_{b, h}^{\sigma}\right)^{2}, \\
\chi_{b}^{\sigma}=\sum_{0 \leq h<k<b}\left(\varphi_{b, h}^{\sigma}-\varphi_{b, k}^{\sigma}\right)^{2} \quad \text { et } \quad \psi_{b}^{\sigma}=\sup _{0 \leq h<k<b}\left|\varphi_{b, h}^{\sigma}-\varphi_{b, k}^{\sigma}\right| .
\end{gathered}
$$

Remarque. Notons que l'on a

$$
\chi_{b}^{\sigma}=b \phi_{b}^{\sigma}-\left(\varphi_{b}^{\sigma}\right)^{2} \quad \text { et } \quad \psi_{b}^{\sigma}=\sup _{0 \leq h<b} \varphi_{b, h}^{\sigma}+\sup _{0 \leq h<b}\left(-\varphi_{b, h}^{\sigma}\right) .
$$

\subsection{Premières propriétés des suites $S_{B}^{\Sigma}$}

Notations. Pour alléger les écritures nous noterons souvent $x_{i}=S_{B}^{\Sigma}(i)$, $y_{i}=S_{B}^{I}(i), X=S_{B}^{\Sigma}, Y=S_{B}^{I}, X_{n}=\left(x_{1}, \ldots, x_{B_{n}}\right)$ et $Y_{n}=\left(y_{1}, \ldots, y_{B_{n}}\right)$; nous désignerons par $\bar{X}_{n}$ le support de $X_{n}$ et par $\bar{Y}_{n}$ celui de $Y_{n}$.

PropriÉté 3.1. Soient $n, i, k$ trois entiers tels que

$$
1 \leq i \leq B_{n-1} \quad \text { et } \quad 0 \leq k \leq b_{n}-1
$$

alors

$$
x_{k B_{n-1}+i}=x_{i}+\left(\sigma_{n-1}(k)-\sigma_{n-1}(0)\right) / B_{n} .
$$

Preuve. Si $i-1=\sum_{j=0}^{n-2} a_{j}(i) B_{j}$, on a

$$
x_{i}=\sum_{j=0}^{n-2} \sigma_{j}\left(a_{j}(i)\right) / B_{j+1}+\sum_{j=n-1}^{+\infty} \sigma_{j}(0) / B_{j+1}
$$

et

$$
x_{k B_{n-1}+i}=\sum_{j=0}^{n-2} \sigma_{j}\left(a_{j}(i)\right) / B_{j+1}+\sigma_{n-1}(k) / B_{n}+\sum_{j=n}^{+\infty} \sigma_{j}(0) / B_{j+1},
$$

d'où le résultat.

PropriÉTÉ 3.2. Soient un entier $n \geq 1$ et $u, v$ appartenant à $\bar{Y}_{n-1}$ tels que $v-u=1 / B_{n-1}$; alors $\left[u, v\left[\right.\right.$ contient $b_{n}$ termes de $X_{n}$ dont un seul est terme de $X_{n-1}$; si ce dernier terme est d'indice $i\left(1 \leq i \leq B_{n-1}\right)$, alors les $b_{n}$ termes de $X_{n}$ dans $\left[u, v\right.$ [ ont pour indice $k B_{n-1}+i$ où $0 \leq k \leq b_{n}-1$.

Preuve. Cette propriété résulte du fait que les éléments de $\bar{X}_{n}$ constituent les sommets d'un polygone régulier de $B_{n}$ côtés inscrit dans $[0,1[$ identifié au tore et de la propriété 3.1.

Remarque. En ordonnant l'ensemble des $\sigma_{n-1}(k)$ pour $0 \leq k<b_{n}$ :

$$
0=\sigma_{n-1}\left(k_{0}\right)<\sigma_{n-1}\left(k_{1}\right)<\ldots<\sigma_{n-1}\left(k_{b_{n}-1}\right)=b_{n}-1,
$$


on a

$$
u \leq x_{k_{0} B_{n-1}+i}<x_{k_{1} B_{n-1}+i}<\ldots<x_{k_{b_{n}-1} B_{n-1}+i}<v .
$$

L'ordre des points de $X_{n}$ dans $[u, v$ [ est donc le même que celui des points de $\mathcal{Z}_{b_{n}}^{\sigma_{n-1}}$ dans $[0,1[$.

\subsection{Propriétés des fonctions associées à un couple $(b, \sigma)$}

PROPRIÉtÉ 3.3. Les fonctions $\varphi_{b, h}^{\sigma}(0 \leq h<b), \varphi_{b}^{\sigma}$ et $\psi_{b}^{\sigma}$ sont affines par morceaux et continues; les fonctions $\phi_{b}^{\sigma}$ et $\chi_{b}^{\sigma}$ sont paraboliques par morceaux et continues.

La démonstration pour $\varphi_{b, h}^{\sigma}$ est analogue à celle des propriétés 3.2 de [6]; les propriétés pour les autres fonctions s'en déduisent immédiatement.

PropriÉtÉ 3.4. Les coefficients des fonctions $\varphi_{b, h}^{\sigma}$ sont des entiers de valeur absolue au plus égale à $b-1$; de plus,

$$
\left|\varphi_{b, h}^{\sigma}\right| \leq b / 4 \quad \text { et } \quad\left(\varphi_{b, h}^{\sigma}\right)^{\prime}(k / b)=\left(\varphi_{b, h}^{I}\right)^{\prime}(\sigma(k) / b)
$$

où $k$ est un entier $(0 \leq k \leq b-1)$ et où par convention $f^{\prime}$ désigne la dérivée à droite de $f$.

Ces propriétés résultent directement de la définition des fonctions $\varphi_{b, h}^{\sigma}$ et, pour $\left|\varphi_{b, h}^{\sigma}\right| \leq b / 4$, de la propriété 3.2 .2 de [6].

PropriétÉ 3.5. (i) La fonction $\varphi_{b}^{\sigma}=\sum_{h=1}^{b-1} \varphi_{b, h}^{\sigma}$ vérifie

$$
\left(\varphi_{b}^{\sigma}\right)^{\prime}((k-1) / b)=b((b-1) / 2-\sigma(k-1)) \quad \text { pour } k=1, \ldots, b ;
$$

par suite, on peut reconstituer $\sigma$ avec la donnée de $\varphi$. En outre, on a

$$
\varphi_{b}^{\sigma}(k / b)=\sum_{j=1}^{k}\left(\varphi_{b}^{\sigma}\right)^{\prime}((j-1) / b) / b .
$$

(ii) Sur chaque intervalle $[(k-1) / b, k / b]$, les arcs de parabole constituant le graphe de $\chi_{b}^{\sigma}$ sont tous translatés d'arcs de la parabole $y=b^{2}\left(b^{2}-1\right) x^{2} / 12$; en particulier,

$$
\chi_{b}^{\sigma}(x)=b^{2}\left(b^{2}-1\right) x^{2} / 12 \quad \text { sur }[0,1 / b] .
$$

Preuve. (i) La quantité $\varphi^{\prime}((k-1) / b)$ représente la pente de la fonction $\varphi$ sur $[(k-1) / b, k / b]$; on vérifie facilement que la somme des pentes des fonctions $\varphi_{h}$ donne la formule annoncée. Les pentes de $\varphi$ sont prises une fois et une seule dans l'ensemble des $b((b-1) / 2-k)$ où $k$ varie de 0 à $b-1$; grâce à la continuité de $\varphi$, les pentes sur chaque intervalle permettent donc de reconstituer $\sigma$.

(ii) Rappelons que $\chi=b \phi-\varphi^{2}$ et $\phi=\sum_{h=1}^{b-1}\left(\varphi_{h}\right)^{2}$; il suffit de calculer le coefficient dominant de $\chi$ sur chaque intervalle; on s'aperçoit alors qu'il vaut 
$b^{2}\left(b^{2}-1\right) / 12$. L'expression de $\chi$ sur $[0,1 / b]$ est enfin obtenue en calculant $\chi(1 / b)=\left(b^{2}-1\right) / 12$ puisque $\chi(0)=0$.

\section{Résultats}

\subsection{Formules pour la discrépance et la diaphonie}

ThÉORÈme 4.1. Pour tout entier $N \geq 1$, on a

$$
T^{2}\left(N, S_{B}^{\Sigma}\right)=\sum_{j=1}^{\infty} \phi_{b_{j}}^{\sigma_{j-1}}\left(N / B_{j}\right) / b_{j}+\sum_{i \neq j} \varphi_{b_{i}}^{\sigma_{i-1}}\left(N / B_{i}\right) \varphi_{b_{j}}^{\sigma_{j-1}}\left(N / B_{j}\right) /\left(b_{i} b_{j}\right) \text {. }
$$

ThÉorème 4.2. Pour tout entier $N \geq 1$, on a

$$
F^{2}\left(N, S_{B}^{\Sigma}\right)=4 \pi^{2} \sum_{j=1}^{\infty} \chi_{b_{j}}^{\sigma_{j-1}}\left(N / B_{j}\right) / b_{j}^{2} .
$$

THÉORÈme 4.3. Si I désigne la suite des permutations identiques en base variable $B$, pour tout $N \geq 1$, on a

$$
D^{(1)}\left(N, S_{B}^{I}\right)=\sum_{j=1}^{\infty} \varphi_{b_{j}}^{I}\left(N / B_{j}\right) / b_{j} .
$$

La propriété 2.4 nous a conduit à étudier l'effet d'une translation modulo $b$ sur une permutation $\sigma$ pour la discrépance extrême et la diaphonie de $S_{b}^{\sigma}$; nous obtenons le théorème ci-après.

ThÉORÈme 4.4. Soit $l$ un entier tel que $0<l<b$; étant donnée une permutation $\sigma$, soit $\tau$ la permutation définie par

$$
\tau(k)=\sigma(k)+l(\bmod b) \quad \text { pour } 0 \leq k \leq b-1 ;
$$

on a alors

$$
D\left(N, S_{b}^{\sigma}\right)=D\left(N, S_{b}^{\tau}\right) \quad \text { et } \quad F\left(N, S_{b}^{\sigma}\right)=F\left(N, S_{b}^{\tau}\right) .
$$

Remarque. La suite $S_{b}^{\tau}$ n'est pas translatée modulo 1 de $S_{b}^{\sigma}$ (voir la fin du paragraphe 5.6).

\subsection{Estimations asymptotiques}

4.2.1. Bases variables

ThÉORÈme 4.5. Pour toute base $B$, pour toute suite $\Sigma$ de permutations et pour tout $p$ vérifiant $1 \leq p \leq \infty$, on a

$$
D^{(p)}\left(N, S_{B}^{\Sigma}\right) \in O(\log N) \quad \text { si } \sum_{j=1}^{n} b_{j} \in O(n) ;
$$

plus précisément, si $\sum_{j=1}^{n} b_{j} \leq K n$ et $b=\inf b_{j}$, alors 


$$
D^{(p)}\left(N, S_{B}^{\Sigma}\right) \leq D\left(N, S_{B}^{\Sigma}\right) \leq(K /(4 \log b)) \log N+K / 4+1,
$$

pour tout $N \geq 1$.

ThÉORÈme 4.6. Pour toute base $B$ et toute suite $\Sigma$, on a

$$
F^{2}\left(N, S_{B}^{\Sigma}\right) \in O(\log N) \quad \text { si } \sum_{j=1}^{n} b_{j}^{2} \in O(n) ;
$$

plus précisément, si $\sum_{j=1}^{n} b_{j}^{2} \leq K n$ et $b=\inf b_{j}$, alors

$$
F^{2}\left(N, S_{B}^{\Sigma}\right)<(C / \log b) \log N+C+\pi^{2} / 3 \quad \text { où } C=(K-b) \pi^{2} / 4 .
$$

Remarques. 1. Dans les deux théorèmes précédents les conditions suffisantes sur la base $B$ ne sont pas nécessaires (voir les familles de contreexemples données au paragraphe 4.4).

2. Notre constante du théorème 4.6 améliore un résultat de Proinov [13] qui obtient $C=(K-1) \pi^{2} / 3$.

3. Le théorème 4.6 et la formule de Koksma impliquent

$$
T^{2}\left(N, S_{B}^{\Sigma}\right)=\left(\sum_{j=1}^{\infty} \varphi_{b_{j}}^{\sigma_{j-1}}\left(N / B_{j}\right) / b_{j}\right)^{2}+O(\log N) \quad \text { si } \sum_{j=1}^{n} b_{j}^{2} \in O(n) .
$$

Cette dernière condition avait été oubliée dans la deuxième formule du théorème 1 de $[3]$.

ThÉORÈme 4.7. Pour toute base $B$ et pour tout $p$ vérifiant $1 \leq p \leq \infty$, on $a$

$$
D^{(p)}\left(N, S_{B}^{I}\right) \in O(\log N) \text { si et seulement si } \sum_{j=1}^{n} b_{j} \in O(n) .
$$

ThÉORÈme 4.8. Pour toute base $B$, on a

$$
F^{2}\left(N, S_{B}^{I}\right) \in O(\log N) \text { si et seulement si } \sum_{j=1}^{n} b_{j}^{2} \in O(n) \text {. }
$$

Remarques. 1. Les théorèmes 4.7 et 4.8 ont été montrés par Proinov et Atanassov ([1], [13] et [14]); nous les retrouvons ici avec de meilleures constantes explicites.

2. Rappelons que pour toute suite $X$, on a $F^{2}(N, X) \in \Omega(\log N)$ d'après [12] et $T^{2}(N, X) \in \Omega(\log N)$ d'après K. F. Roth ([9], p. 105).

4.2.2. Bases fixes

THÉORÈME 4.9. Si $\Sigma=(\sigma)$ est une suite constante, en posant

$$
\beta_{b}^{\sigma}=\inf _{n \geq 1} \sup _{x \in \mathbb{R}}\left|\sum_{j=1}^{n} \varphi_{b}^{\sigma}\left(x / b^{j}\right) / n\right|
$$


on $a$

$$
t_{1}\left(S_{b}^{\sigma}\right)=\limsup _{N \rightarrow+\infty}\left(T\left(N, S_{b}^{\sigma}\right) / \log N\right)=\beta_{b}^{\sigma} /(b \log b) .
$$

ThÉORÈme 4.10. Si $\Sigma=(\sigma)$ est une suite constante, en posant

$$
\gamma_{b}^{\sigma}=\inf _{n \geq 1} \sup _{x \in \mathbb{R}}\left(\sum_{j=1}^{n} \chi_{b}^{\sigma}\left(x / b^{j}\right) / n\right)
$$

on $a$

$$
f\left(S_{b}^{\sigma}\right)=\limsup _{N \rightarrow+\infty}\left(F^{2}\left(N, S_{b}^{\sigma}\right) / \log N\right)=4 \pi^{2} \gamma_{b}^{\sigma} /\left(b^{2} \log b\right) .
$$

4.2.3. Application aux suites symétriques. Une suite $Y=\left(y_{n}\right)$ est dite symétrique si, pour tout $n$, on a

$$
y_{2 n}+y_{2 n+1}=1 .
$$

Une suite symétrique $Y=\left(y_{n}\right)$ est dite produite par la suite $X=\left(x_{n}\right)$ si, pour tout $n$, on a $y_{2 n}=x_{n}$ ou $y_{2 n+1}=x_{n}$; on note alors $Y=\widetilde{X}$.

Pour toute suite symétrique $Y$, on a la relation

$$
\left|\int_{0}^{1} E(\alpha, N, Y) d \alpha\right|=\left|\sum_{n=1}^{N}\left(1 / 2-y_{n}\right)\right| \leq 1 / 2
$$

grâce à la formule de Koksma (voir la propriété 2.2), on en déduit la propriété (théorème $\mathrm{A},[15]$ )

$$
T^{2}(N, Y) \leq(F([N / 2], X) / \pi+a)^{2}+b^{2}
$$

où $a$ et $b$ sont deux constantes, ce qui permet d'obtenir le théorème suivant.

ThÉORÈme 4.11. Pour toute suite $S_{B}^{\Sigma}$ vérifiant $\sum_{j=1}^{n} b_{j}^{2} \in O(n)$, on a

$$
t_{2}\left(\widetilde{S}_{B}^{\Sigma}\right)=\limsup _{N \rightarrow+\infty}\left(T^{2}\left(N, \widetilde{S}_{B}^{\Sigma}\right) / \log N\right) \leq f\left(S_{B}^{\Sigma}\right) / \pi^{2}
$$

et, pour tout $N$,

$$
T^{2}\left(N, \widetilde{S}_{B}^{\Sigma}\right) \leq 4 \sum_{j=0}^{\infty} \chi_{b_{j}}^{\sigma_{j-1}}\left([N / 2] / B_{j}\right) / b_{j}^{2}+O(\sqrt{\log N}) .
$$

Remarque. L'intérêt des suites symétriques pour la discrépance quadratique $T$ a été remarqué pour la première fois par Proinov [11]; il a obtenu ainsi l'ordre exact de $T$ pour les suites infinies en dimension 1, à savoir $\sqrt{\log N}$. Le problème est à présent d'obtenir la meilleure constante possible pour cet ordre de croissance (voir le théorème 4.17).

4.3. Etude détaillée de cas particuliers en base fixe. Nous traitons d'abord le cas des suites de van der Corput-Halton pour lesquelles nous obtenons les limites supérieures exactes relatives à la discrépance $D^{(p)}$ (pour $1 \leq p \leq 2$ ) et à la diaphonie $F$. 
Nous déterminons ensuite, pour chaque base, des permutations optimales pour la discrépance quadratique $T$ des suites $S_{b}^{\sigma}$.

En ce qui concerne la diaphonie, nous avons d'abord trouvé au moyen de calculs sur machine les meilleures permutations (actuelles) pour $2 \leq b \leq 11$; ces calculs nous ont suggéré un type de bonnes permutations pour $b$ impair, le cas $b=19$ s'est avéré être le meilleur de notre étude numérique. Nous signalons enfin les retombées de ces derniers résultats pour les suites symétriques associées.

ThÉorÈme 4.12. Dans le cas des suites de van der Corput-Halton, en posant

$$
d^{(p)}\left(S_{b}^{I}\right)=\limsup _{N \rightarrow+\infty}\left(D^{(p)}\left(N, S_{b}^{I}\right) / \log N\right),
$$

on obtient, pour $p$ réel $(1 \leq p \leq 2)$,

$$
d^{(p)}\left(S_{b}^{I}\right)= \begin{cases}\left(b^{2}+b-2\right) /(8(b+1) \log b) & \text { si } b \text { est pair }, \\ \left(b^{2}-1\right) /(8 b \log b) & \text { si } b \text { est impair. }\end{cases}
$$

R e m a rqu e. Ce théorème a été annoncé par Proinov et Atanassov dans leur note aux Comptes Rendus [14]; nous le retrouvons ici par nos propres méthodes.

ThÉORÈme 4.13. Dans le cas des suites de van der Corput-Halton, en posant

on $a$

$$
f\left(S_{b}^{I}\right)=\limsup _{N \rightarrow+\infty}\left(F^{2}\left(N, S_{b}^{I}\right) / \log N\right),
$$

$$
f\left(S_{b}^{I}\right)= \begin{cases}\pi^{2}\left(b^{3}+b^{2}+4\right) /(48(b+1) \log b) & \text { si b est pair } \\ \pi^{2}\left(b^{4}+2 b^{2}-3\right) /\left(48 b^{2} \log b\right) & \text { si b est impair }\end{cases}
$$

Rem arque. Le résultat de ce théorème rend caduques les majorations obtenues par Proinov et Grozdanov [16] :

$$
f\left(S_{b}^{I}\right) \leq \pi^{2}\left(b^{2}-1\right) /(3 \log b),
$$

Xiao [18] :

$$
f\left(S_{b}^{I}\right) \leq \pi^{2}(b+1)^{2}\left(b^{2}-1\right) /\left(12 b^{2} \log b\right)
$$

et Pagès [10] :

$$
f\left(S_{b}^{I}\right) \leq \pi^{2}\left(b^{2}-1\right) /(12 \log b) .
$$

ThÉorÈme 4.14. Dans la famille des suites $S_{b}^{\sigma}$, les meilleures permutations pour la discrépance quadratique sont:

- dans le cas b pair, la permutation $\sigma_{0}$ définie par

$$
\sigma_{0}(i)= \begin{cases}b / 2-(i+1) & \text { pour } i \text { pair }(0 \leq i<b / 2), \\ b / 2+i & \text { pour } i \text { impair }(0 \leq i<b / 2),\end{cases}
$$




$$
\sigma_{0}(i)+\sigma_{0}(b-i-1)=b-1,
$$

cette permutation donne l'estimation

$$
1 /(8 \log b) \leq t_{1}\left(S_{b}^{\sigma_{0}}\right) \leq(b+1) /(8 b \log b) ;
$$

- dans le cas $b$ impair, la permutation $\sigma_{0}$ définie par

$$
\begin{gathered}
\sigma_{0}(i)= \begin{cases}(b-1) / 2-(i+1) & \text { pour } i \text { pair }(0 \leq i<(b-1) / 2), \\
(b+1) / 2+i & \text { pour } i \text { impair }(0 \leq i<(b-1) / 2),\end{cases} \\
\sigma_{0}(i)+\sigma_{0}(b-2-i)=b-1 \quad \text { et } \quad \sigma_{0}(b-1)=(b-1) / 2,
\end{gathered}
$$

cette permutation donne l'estimation

$$
1 /(8 \log b) \leq t_{1}\left(S_{b}^{\sigma_{0}}\right) \leq(b+4) /(8 b \log b) .
$$

Remarques. 1. La constante $t_{1}\left(S_{b}^{\sigma_{0}}\right)$ tend donc vers zéro alors que $t_{1}\left(S_{b}^{I}\right)=d^{(2)}\left(S_{b}^{I}\right)$ tend vers l'infini quand $b$ tend vers l'infini (voir théorème 4.12); cela n'est pas surprenant car l'ordre exact de $T$ est $\sqrt{\log N}$. On voit bien ici l'intérêt d'introduire des permutations pour les discrépances.

2. Notons que les permutations $w$ construites par Braaten et Weller [2] peuvent aussi être étudiées par notre méthode à l'aide des fonctions $\varphi$ associées. Cette étude montre que

$$
(b-3) /(2 b \log b) \leq t_{1}\left(S_{b}^{w}\right) \leq(b-1) /(2 b \log b) .
$$

ThÉORÈme 4.15. Pour $2 \leq b \leq 11$, la plus faible diaphonie est obtenue pour les permutations données dans le tableau suivant:

\begin{tabular}{cccc}
\hline$b$ & permutation $\sigma$ & minorant de $f\left(S_{b}^{\sigma}\right)$ & majorant de $f\left(S_{b}^{\sigma}\right)$ \\
\hline 2 & $(01)$ & $1.58209 \cdots$ & $1.58209 \cdots$ \\
3 & $(012)$ & $1.99637 \cdots$ & $1.99637 \cdots$ \\
4 & $(0213)$ & $1.58209 \cdots$ & $1.58209 \cdots$ \\
5 & $(03124)$ & $1.59440 \cdots$ & 1.667 \\
6 & $(042135)$ & $1.74867 \cdots$ & 1.824 \\
7 & $(0351426)$ & $1.42613 \cdots$ & 1.479 \\
8 & $(03516247)$ & $1.51146 \cdots$ & 1.636 \\
9 & $(053716248)$ & $1.37528 \cdots$ & 1.451 \\
10 & $(0472851639)$ & $1.33525 \cdots$ & 1.433 \\
11 & $(0735918426 \overline{10})$ & $1.43887 \cdots$ & 1.592 \\
\hline
\end{tabular}

Remarques. 1. Ces permutations ont été obtenues par recherche systématique à la machine, recherche facilitée du fait qu'il suffit de considérer les permutations $\sigma$ telles que $\sigma(0)=0$ d'après le théorème 4.4. En fait, pour chaque base nous trouvons plusieurs permutations donnant le même résultat.

2. Les résultats pour $b=2,3$ et 4 sont les valeurs exactes de $f\left(S_{b}^{\sigma}\right)$. Pour les autres valeurs de $b$ les minorants sont probablement les valeurs exactes de $f\left(S_{b}^{\sigma}\right)$; nous n'avons pas jugé utile de mener au bout ces calculs, car nous avons un meilleur résultat pour $b=19$ (voir le théorème 4.16). 
3. Les permutations obtenues pour $b$ impair inférieur à 11 suggèrent une famille de permutations donnant, pour toute base impaire, une très faible diaphonie; ces familles sont construites selon la règle suivante : $\sigma(0)=0$, $\sigma((b-1) / 2)=1, \sigma(b-1)=b-1, \sigma(i)$ impair pour $1 \leq i<(b-1) / 2$ et $\sigma(b-i-1)=\sigma(i)-1$.

Le meilleur résultat pour $13 \leq b \leq 21$ est obtenu avec $b=19$; il constitue la plus faible diaphonie actuellement connue, pour cette raison nous avons mené le calcul à son terme, consigné dans le théorème suivant.

THÉORÈME 4.16. En base 19 et pour la permutation

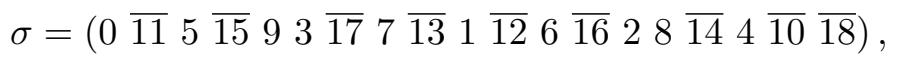

on obtient

$$
f\left(S_{19}^{\sigma}\right)=\frac{4 \pi^{2} \gamma_{19}^{\sigma}}{19^{2} \log 19}=\frac{3826 \pi^{2}}{27 \times 19^{2} \log 19}=1.31574 \ldots
$$

Remarques. 1. Pour les autres bases impaires entre 13 et 21, les permutations obtenues par la même règle donnent pour minorant de $f\left(S_{b}^{\sigma}\right)$ respectivement $1.35 \ldots, 1.40 \ldots, 1.33 \ldots$ et $1.36 \ldots$ qui sont probablement les valeurs exactes.

2. Les théorèmes 4.15 et 4.16 améliorent les résultats annoncés dans [2].

ThÉORÈme 4.17. Avec les couples $(b, \sigma)$ des théorèmes 4.15 et 4.16 , on obtient les majorations suivantes pour la discrépance quadratique des suites symétriques $\widetilde{S}_{b}^{\sigma}$ (voir 4.2.3) :

\begin{tabular}{cccccccccccc}
\hline$b$ & 2 & 3 & 4 & 5 & 6 & 7 & 8 & 9 & 10 & 11 & 19 \\
\hline $\begin{array}{l}\text { majorant } \\
\text { de } t_{2}\left(\widetilde{S}_{b}^{\sigma}\right)\end{array}$ & .161 & .203 & .161 & .169 & .185 & .150 & .166 & .148 & .146 & .162 & .134 \\
\hline
\end{tabular}

R e marques. 1. En base 2, l'étude directe de l'écart de la suite de van der Corput symétrique a permis un encadrement très précis :

$$
.089<t_{2}\left(\widetilde{S}_{2}^{I}\right)<.103 \quad \text { (H. Faure [7]) }
$$

le majorant est la meilleure constante actuellement connue pour la discrépance quadratique.

2. La majoration du théorème 4.11 et les constantes du théorème 4.17 qui en résultent montrent les limites de l'approche de $t_{2}\left(\widetilde{S}_{b}^{\sigma}\right)$ par $f\left(S_{b}^{\sigma}\right)$; il faut une étude spécifique directe des suites symétriques en base $b$ quelconque pour espérer faire mieux que pour la base 2 .

4.4. Familles de contre-exemples pour la discrépance et la diaphonie en base variable. Signalons que les résultats obtenus ici résultent 
d'un algorithme décrit par H. Faure [8], permettant de construire des suites $\Sigma_{0}=\left(\sigma_{j-1}\right)_{j}$ de permutations telles que

$$
d_{b_{j}}^{\sigma_{j-1}} \leq \log b_{j} / \log 2 \quad \text { pour tout } j \geq 1,
$$

où $d_{b}^{\sigma}=\max \psi_{b}^{\sigma}$ et $\psi_{b}^{\sigma}=\max _{h} \varphi_{b, h}^{\sigma}-\min _{h} \varphi_{b, h}^{\sigma}$ et où $\sigma_{j-1}$ est déterminée à partir de $b_{j}$ par une correspondance dont la construction est la suivante :

- Pour $b=2$, prendre $\sigma=I$;

- Supposons toutes les permutations construites pour $b=2,3, \ldots, b^{\prime}-1$; si $b^{\prime}=2 c$, prendre $\sigma$ définie par

$$
\sigma(l)=2 \tau(h)+k
$$

où $l=k c+h, 0 \leq h \leq c-1,0 \leq k \leq 1$ et où $\tau$ est la permutation déjà construite pour la base $c$; si $b^{\prime}=2 c+1$, prendre $\sigma^{\prime}$ définie par

$$
\begin{aligned}
& \sigma^{\prime}(c)=c, \\
& \sigma^{\prime}(k)= \begin{cases}\sigma(k) & \text { pour } 0 \leq \sigma(k)<c \text { et } 0 \leq k<c, \\
\sigma(k+1) & \text { pour } c \leq \sigma(k)<2 c \text { et } 0 \leq k<c, \\
\sigma(k-1) & \text { pour } 0 \leq \sigma(k-1)<c \text { et } c<k \leq 2 c, \\
\sigma(k-1)+1 & \text { pour } c \leq \sigma(k-1)<2 c \text { et } c<k \leq 2 c\end{cases}
\end{aligned}
$$

où $\sigma$ est la permutation déjà construite pour la base $2 c$.

Pour les premières valeurs de $b$, on obtient la correspondance :

$$
\begin{array}{cccccccc}
b & 2 & 3 & 4 & 5 & 6 & 7 & 8 \\
\sigma & (01) & (012) & (0213) & (03214) & (024135) & (0253146) & (04261537)
\end{array}
$$

THÉORÈme 4.18. Soit $B=\left(b_{j}\right)$ une base variable vérifiant les deux hypothèses " $\sum_{j=1}^{n} b_{j} \notin O(n)$ " et "il existe $K>0$ tel que, pour tout entier $n \geq 2, b_{n} \leq\left(\prod_{i=1}^{n-1} b_{i}\right)^{K} " ;$ alors il existe des suites $\Sigma_{0}$ de permutations telles que, pour $N \geq 1$,

$$
D\left(N, S_{B}^{\Sigma_{0}}\right) \leq(K+1) \log N / \log 2+1 .
$$

R e marque. Ce théorème, démontré pour la discrépance $D$, est encore valable pour les discrépances $D^{*}$ et $T$ en vertu des inégalités

$$
T\left(N, S_{B}^{\Sigma}\right) \leq D^{*}\left(N, S_{B}^{\Sigma}\right) \leq D\left(N, S_{B}^{\Sigma}\right) .
$$

Exemples. Nous donnons ici des exemples de base variable $B=$ $\left(b_{j}\right)_{j \geq 1}$ vérifiant les hypothèses du théorème 4.18.

1. Avec $b_{1}=2$ et $b_{j}=j$ pour $j \geq 2$, on obtient $\sum_{j=1}^{n} b_{j} \sim n^{2} / 2$ et on peut prendre $K=1$.

2. Soient deux entiers $b \geq 2$ et $c \geq 1$; avec $b_{1}=b^{2 c}$ et $b_{j}=b^{j c}$ pour $j \geq 2$, on obtient $\sum_{j=1}^{n} b_{j} \sim b^{c(n+1)} /\left(b^{c}-1\right)$ et on peut prendre $K=1$.

Remarquons que Atanassov [1] a donné comme exemple $B=\left(2^{\alpha_{n}}\right)$ avec $\alpha_{n}$ une suite d'entiers positifs, mais sa suite de permutations $\Sigma$ est telle que 
$S_{B}^{\Sigma}$ coïncide avec la suite $S_{2}^{I}$ de van der Corput en base 2 . Il en est de même dans notre exemple 2 avec $b$ puissance de 2 ; ce qui est dû à la construction des permutations associées aux bases puissance de 2 dans l'algorithme précité où seule la première partie est utilisée; par contre, si $b$ n'est pas une puissance de 2 , on ne retrouve pas la suite $S_{b}^{I}$.

ThÉORÈme 4.19. Soit $B=\left(b_{j}\right)$ une base variable vérifiant les deux hypothèses " $\sum_{j=1}^{n} b_{j}^{2} \notin O(n)$ " et "il existe $K>0$ tel que, pour tout entier $n \geq 1, \sum_{j=1}^{n}\left(\log b_{j}\right)^{2} \leq K n " ;$ alors il existe des suites $\Sigma_{0}$ de permutations telles que, pour $N \geq 1$,

$$
F^{2}\left(N, S_{B}^{\Sigma_{0}}\right) \leq 2 \pi^{2}(\log 2)^{-2} K(\log N / \log b+1)+\pi^{2} / 3 .
$$

Remarques. 1. Plus simplement ce théorème donne

$$
f\left(S_{B}^{\Sigma_{0}}\right) \leq 2 \pi^{2} K(\log 2)^{-2}(\log b)^{-1}
$$

et, d'après la propriété rappelée en 4.2 .3 , il en résulte

$$
t_{2}\left(\widetilde{S}_{B}^{\Sigma_{0}}\right) \leq 2 K(\log 2)^{-2}(\log b)^{-1} .
$$

2. Rappelons que ce résultat est aussi un contre-exemple pour la réciproque d'un théorème de Proinov sur la diaphonie ([13], théorème 1).

Exemple. Soit $B=\left(b_{j}\right)_{j \geq 1}$ définie par $b_{1}=b, b_{j}=b$ si $j$ est non carré, $b_{j}=\sqrt{j}$ si $j$ est un carré différent de 1 , où $b$ est un entier strictement supérieur à 1 ; cette base vérifie les propriétés

$$
\sum_{j=1}^{n} b_{j}^{2} \sim n^{3 / 2} / 3 \quad \text { et } \quad \sum_{j=1}^{n}\left(\log b_{j}\right)^{2} \leq 3(\log b)^{2} n .
$$

$\mathrm{Si}$, dans la conclusion du théorème 4.19 , on se contente de la propriété pour $N$ assez grand, on peut ramener la constante $K$ de cet exemple à $(\log b)^{2}+\varepsilon$.

5. Démonstration des résultats du paragraphe 4.1. Nous utiliserons les notations et propriétés des paragraphes 3.2 et 3.3 .

5.1. Lemme De Discrétisation. Soient $n$ et $N$ deux entiers vérifiant $1 \leq N \leq B_{n}$ et $\alpha$ un réel de $[0,1]$; on a alors

$$
E\left(\alpha, N, S_{B}^{\Sigma}\right)=E\left(y(\alpha), N, S_{B}^{\Sigma}\right)+(y(\alpha)-\alpha) N
$$

où $y(\alpha)$ est défini comme suit : il existe $u$ et $v$ uniques de $\bar{Y}_{n}$ tels que $u \leq \alpha<v$ et $v-u=B_{n}^{-1}$ et il existe $x$ unique de $\bar{X}_{n}$ tel que $u \leq x<v$; cela étant, $y(\alpha)=u$ si $\alpha \leq x$ et $y(\alpha)=v$ si $\alpha>x$.

Preuve. Le choix de $y(\alpha)$ entraîne que $A(\alpha, N, X)=A(y(\alpha), N, X)$, d'où le résultat. 
5.2. Lemme De DeSCEnTE. Soient $n$ et $N$ deux entiers vérifiant $1 \leq$ $N \leq B_{n}$, soient $\lambda$ un entier tel que $0 \leq \lambda<B_{n}$ et $\sum_{i=1}^{n} \lambda_{i} B_{n} B_{i}^{-1}$ son développement en base variable $B$; alors

$$
E\left(\lambda / B_{n}, N, S_{B}^{\Sigma}\right)=\sum_{j=1}^{n} \varphi_{b_{j}, \varepsilon_{j}}^{\sigma_{j-1}}\left(N / B_{j}\right)
$$

où les $\varepsilon_{j}$ sont $n$ entiers définis de proche en proche comme suit : $\varepsilon_{n}=\delta_{n}=$ $\lambda_{n}$ et, si $1 \leq j<n$,

$$
\begin{gathered}
\delta_{j}=\lambda_{j}+\delta_{j+1} / b_{j+1}+\left(\varphi_{b_{j+1}, \varepsilon_{j+1}}^{\sigma_{j}}\right)^{\prime}\left(N / B_{j+1}\right) / b_{j+1}, \\
\varepsilon_{j}=\delta_{j} \quad \text { si } 0 \leq \delta_{j}<b_{j} \quad \text { et } \quad \varepsilon_{j}=0 \quad \text { si } \delta_{j}=b_{j} .
\end{gathered}
$$

Preuve. La construction de $\varepsilon_{j}$ résulte de la démonstration suivante qui s'effectue par récurrence de $j=n$ à $j=1$.

Le procédé consiste à écrire l'écart sur des intervalles de plus en plus grossiers avec des suites ayant de moins en moins de points; à chaque étape, le passage d'un intervalle à un intervalle plus grossier fait apparaître les valeurs des dérivées des fonctions $\varphi_{b, h}^{\sigma}$, et la différence entre les écarts sur ces intervalles fait apparaître les valeurs de ces mêmes fonctions.

Le cas $N=B_{n}$ est trivial car $N / B_{j}$ est entier et $A\left(\lambda / B_{n}, N\right)=\lambda$.

Pour $N<B_{n}$, on note $N=\sum_{i=0}^{n-1} N_{i} B_{i}$ (développement en base $B$ ).

Etape 1 : Posons $y=\lambda / B_{n} ; y$ appartient à $\bar{Y}_{n}$, il existe donc $u$ et $v$ uniques dans $\bar{Y}_{n-1}$ tels que $u \leq y<v$ et $v-u=1 / B_{n-1}$. D'après la propriété 3.2 , il existe un indice $i$ unique tel que $x_{i}$ appartienne à $\bar{X}_{n-1} \cap$ $\left[u, v\left[\right.\right.$ et de plus $x_{i+N_{n-1} B_{n-1}}$ appartienne à $\bar{X}_{n} \cap[u, v[$.

Si $y \leq x_{i+N_{n-1} B_{n-1}}$, en posant $y^{\prime}=u$, on a

$$
E\left(y, N, X_{n}\right)=E\left(y^{\prime}, N, X_{n}\right)+A\left(\left[y^{\prime}, y\left[; N ; X_{n}\right)-N\left(y-y^{\prime}\right) ;\right.\right.
$$

l'ordre des points de $X_{n}$ dans [u,v[ étant le même que celui des points de $\mathcal{Z}_{b_{n}}^{\sigma_{n-1}}$ dans $[0,1[$ (propriété 3.2 ), on a

$A\left(\left[y^{\prime}, y\left[; N ; X_{n}\right)=A\left(\lambda_{n} / b_{n}, N_{n-1}+1, \mathcal{Z}_{b_{n}}^{\sigma_{n-1}}\right) \quad\right.\right.$ car $\quad\left(y-y^{\prime}\right) B_{n-1}=\lambda_{n} / b_{n} ;$ nous obtenons alors, avec $\varepsilon_{n}=\lambda_{n}$,

$$
\begin{array}{r}
E\left(y, N, X_{n}\right)=E\left(y^{\prime}, N, X_{n}\right)+\varphi_{b_{n}, \varepsilon_{n}}^{\sigma_{n-1}}\left(N / B_{n}\right) \\
\text { puisque } 0 \leq \lambda_{n} \leq \sigma_{n-1}\left(N_{n-1}\right)
\end{array}
$$

(ce qui se vérifie en utilisant le développement de $x_{i}$ et la propriété 3.1). De plus, en posant $\lambda^{\prime}=y^{\prime} B_{n-1}$, on constate que

$$
\lambda^{\prime}=\left(\lambda+\left(\varphi_{b_{n}, \varepsilon_{n}}^{\sigma_{n-1}}\right)^{\prime}\left(N / B_{n}\right)\right) / b_{n} \text { puisque }\left(\varphi_{b_{n}, \varepsilon_{n}}^{\sigma_{n-1}}\right)^{\prime}\left(N / B_{n}\right)=-\varepsilon_{n} .
$$

Si $x_{i+N_{n-1} B_{n-1}}<y$, en posant $y^{\prime}=v$, on a

$$
A\left(\left[y, y^{\prime}\left[; N ; X_{n}\right)=A\left(\left[\lambda_{n} / b_{n}, 1\left[; N_{n-1}+1 ; \mathcal{Z}_{b_{n}}^{\sigma_{n-1}}\right)\right.\right.\right.\right.
$$


pour les mêmes raisons que précédemment, d'où

$$
\begin{array}{r}
E\left(y, N, X_{n}\right)=E\left(y^{\prime}, N, X_{n}\right)+\varphi_{b_{n}, \varepsilon_{n}}^{\sigma_{n-1}}\left(N / B_{n}\right) \\
\text { puisque } \sigma_{n-1}\left(N_{n-1}\right)<\lambda_{n}<b_{n} .
\end{array}
$$

De nouveau, en posant $\lambda^{\prime}=y^{\prime} B_{n-1}$, on a

$$
\lambda^{\prime}=\left(\lambda+\left(\varphi_{b_{n}, \varepsilon_{n}}^{\sigma_{n-1}}\right)^{\prime}\left(N / B_{n}\right)\right) / b_{n}
$$

mais ici avec $\left(\varphi_{b_{n}, \varepsilon_{n}}^{\sigma_{n-1}}\right)^{\prime}\left(N / B_{n}\right)=b_{n}-\varepsilon_{n}$.

Avec $N^{\prime}=N-N_{n-1} B_{n-1}$, nous avons

$$
\begin{aligned}
A\left(y^{\prime}, N, X_{n}\right)= & A\left(y^{\prime}, N_{n-1} B_{n-1}, X_{n}\right) \\
& +\operatorname{card}\left\{j ; N_{n-1} B_{n-1}<j \leq N, x_{j} \in\left[0, y^{\prime}[\}\right.\right. \\
= & A\left(y^{\prime}, N_{n-1} B_{n-1}, X_{n}\right)+A\left(y^{\prime}, N^{\prime}, X_{n-1}\right),
\end{aligned}
$$

d'où $E\left(y^{\prime}, N, X_{n}\right)=E\left(y^{\prime}, N^{\prime}, X_{n-1}\right)$.

Finalement, la première étape fournit les formules

$$
E\left(\lambda / B_{n}, N, X_{n}\right)=E\left(\lambda^{\prime} / B_{n-1}, N^{\prime}, X_{n-1}\right)+\varphi_{b_{n}, \varepsilon_{n}}^{\sigma_{n-1}}\left(N / B_{n}\right)
$$

avec $\varepsilon_{n}=\lambda_{n}$ et $N^{\prime}, \lambda^{\prime}$ définis ci-dessus.

Etape 2: On commence par vérifier que

$$
\lambda^{\prime}=\sum_{i=1}^{n-2} \lambda_{i} B_{n-1} B_{i}^{-1}+\delta_{n-1}
$$

où $\delta_{n-1}=\lambda_{n-1}+\left(\delta_{n}+\left(\varphi_{b_{n}, \varepsilon_{n}}^{\sigma_{n-1}}\right)^{\prime}\left(N / B_{n}\right)\right) / b_{n}$ et $\delta_{n}=\varepsilon_{n}$, puisque $0 \leq \delta_{n-1} \leq$ $b_{n-1}\left(\operatorname{car} 0 \leq \lambda_{n-1}<b_{n-1}\right)$. d'où

Si $\delta_{n-1}=b_{n-1}$, comme $\varepsilon_{n-1}=0, \varphi_{b_{n-1}, \varepsilon_{n-1}}^{\sigma_{n-2}}$ et sa dérivée sont nulles;

$$
E\left(\lambda^{\prime} / B_{n-1}, N^{\prime}, X_{n-1}\right)=E\left(\lambda^{\prime \prime} / B_{n-2}, N^{\prime}, X_{n-1}\right)+\varphi_{b_{n-1}, \varepsilon_{n-1}}^{\sigma_{n-2}}\left(N^{\prime} / B_{n-1}\right)
$$

avec

$$
\lambda^{\prime \prime}=\left(\lambda^{\prime}+\left(\varphi_{b_{n-1}, \varepsilon_{n-1}}^{\sigma_{n-2}}\right)^{\prime}\left(N / B_{n-1}\right)\right) / b_{n-1} .
$$

Si $\delta_{n-1}<b_{n-1}$, tout se passe de la même façon que dans l'étape 1 , où $\lambda_{n}$ est remplacé par $\delta_{n-1}$; le résultat obtenu est le même que dans le cas $\delta_{n-1}=b_{n-1}$ avec $\varepsilon_{n-1}=\delta_{n-1}$.

En posant $N^{\prime \prime}=N^{\prime}-N_{n-2} B_{n-2}$, de même qu'à la fin de l'étape 1, on a

$$
E\left(\lambda^{\prime \prime} / B_{n-2}, N^{\prime}, X_{n-1}\right)=E\left(\lambda^{\prime \prime} / B_{n-2}, N^{\prime \prime}, X_{n-2}\right) .
$$

Enfin, du fait que $\varphi_{b_{n-1}, \varepsilon_{n-1}}^{\sigma_{n-2}}\left(N^{\prime} / B_{n-1}\right)=\varphi_{b_{n-1}, \varepsilon_{n-1}}^{\sigma_{n-2}}\left(N / B_{n-1}\right)$, l'étape 2 donne

$$
\begin{aligned}
& E\left(\lambda / B_{n}, N, X_{n}\right) \\
& \quad=E\left(\lambda^{\prime \prime} / B_{n-2}, N^{\prime \prime}, X_{n-2}\right)+\varphi_{b_{n-1}, \varepsilon_{n-1}}^{\sigma_{n-2}}\left(N / B_{n-1}\right)+\varphi_{b_{n}, \varepsilon_{n}}^{\sigma_{n-1}}\left(N / B_{n}\right) .
\end{aligned}
$$


Les étapes suivantes se déroulent de manière identique à l'étape 2; au bout de $n-1$ opérations, on obtient

$$
E\left(\lambda / B_{n}, N, X_{n}\right)=E\left(\delta_{1} / B_{1}, N_{0}, X_{1}\right)+\sum_{j=2}^{n} \varphi_{b_{j}, \varepsilon_{j}}^{\sigma_{j-1}}\left(N / B_{j}\right),
$$

ce qui fournit le résultat annoncé en remarquant que

$$
E\left(\delta_{1} / B_{1}, N_{0}, X_{1}\right)=\varphi_{b_{1}, \varepsilon_{1}}^{\sigma_{0}}\left(N_{0} / B_{1}\right)=\varphi_{b_{1}, \varepsilon_{1}}^{\sigma_{0}}\left(N / B_{1}\right) ;
$$

en effet, cela est évident si $\delta_{1}=b_{1}\left(\varepsilon_{1}=0\right)$ et sinon $E\left(\delta_{1} / B_{1}, N_{0}, X_{1}\right)=$ $E\left(\delta_{1} / B_{1}, N_{0}, \mathcal{Z}_{b_{1}}^{\sigma_{0}}\right)$ car les suites $X_{1}$ et $\mathcal{Z}_{b_{1}}^{\sigma_{0}}=\left(z_{i}\right)$ sont liées par $x_{i}=z_{i}+\alpha$ où $\alpha=\sum_{j=2}^{+\infty} \sigma_{j}(0) / B_{j+1}\left(0<\alpha<1 / b_{1}\right)$.

5.3. Lemme (où on explicite les $\varepsilon_{j}$ ). Avec les notations du lemme 5.2, pour $j=0, \ldots, n-1$, posons

$$
\begin{gathered}
\Lambda_{j}=\sum_{h=j+1}^{n} \lambda_{h} B_{n} / B_{h} \\
\nu_{j}=\sum_{h=j}^{n-1} \sigma_{h}\left(N_{h}\right) B_{n} / B_{h+1} \quad \text { ò̀ } \quad N=\sum_{h=0}^{n-1} N_{h} B_{h} ;
\end{gathered}
$$

on a alors, pour $j=1, \ldots, n-1$,

$$
\delta_{j}= \begin{cases}\lambda_{j} & \text { si } 0 \leq \Lambda_{j} \leq \nu_{j} \\ \lambda_{j}+1 & \text { si } \nu_{j}<\Lambda_{j}<B_{n} / B_{j}\end{cases}
$$

et

$$
\varepsilon_{j}= \begin{cases}0 & \text { si } 0 \leq \Lambda_{j-1} \leq \nu_{j} \\ p & \text { si } \nu_{j}+(p-1) B_{n} / B_{j}<\Lambda_{j-1} \leq \nu_{j}+p B_{n} / B_{j} \text { pour } 1 \leq p<b_{j} \\ 0 & \text { si } \nu_{j}+\left(b_{j}-1\right) B_{n} / B_{j}<\Lambda_{j-1} \leq B_{n} / B_{j-1}\end{cases}
$$

Remarque. Les relations sont encore valables pour $j=n$, avec la convention $\Lambda_{n}=\nu_{n}=0$.

Preuve. Montrons tout d'abord par récurrence les relations sur les $\delta_{j}$ dans le cas particulier où $\sigma_{j}=I$ pour tout $j$.

Cas $j=n-1$ : Ici

$$
\begin{gathered}
\Lambda_{n-1}=\lambda_{n}=\delta_{n}=\varepsilon_{n}, \quad \nu_{n-1}=N_{n-1} \\
\delta_{n-1}=\lambda_{n-1}+\left(\delta_{n}+\left(\varphi_{b_{n}, \varepsilon_{n}}^{I}\right)^{\prime}\left(N / B_{n}\right)\right) / b_{n} \quad \text { (voir lemme 5.2) }
\end{gathered}
$$

et

$$
N_{n-1} / b_{n} \leq N / B_{n}<\left(N_{n-1}+1\right) / b_{n} .
$$

Si $0 \leq \Lambda_{n-1} \leq \nu_{n-1},\left(\varphi_{b_{n}, \varepsilon_{n}}^{I}\right)^{\prime}\left(N / B_{n}\right)=-\varepsilon_{n}$ car $\varepsilon_{n} \leq I\left(N_{n-1}\right)=N_{n-1}$ (voir la définition 3.3); d'où $\delta_{n-1}=\lambda_{n-1}$. 
Si $\nu_{n-1}<\lambda_{n-1}<b_{n},\left(\varphi_{b_{n}, \varepsilon_{n}}^{I}\right)^{\prime}\left(N / B_{n}\right)=b_{n}-\varepsilon_{n} \operatorname{car} I\left(N_{n-1}\right)=N_{n-1}<$ $\varepsilon_{n}$ (voir la définition 3.3); d'où $\delta_{n-1}=\lambda_{n-1}+1$. Ici

Cas $1 \leq j<n-1$ : Supposons les relations sur les $\delta$ vraies au rang $j+1$.

$$
\begin{gathered}
\Lambda_{j}=\Lambda_{j+1}+\lambda_{j+1} B_{n} / B_{j+1}, \quad \nu_{j}=\nu_{j+1}+N_{j} B_{n} / B_{j+1}, \\
\delta_{j}=\lambda_{j}+\left(\delta_{j+1}+\left(\varphi_{b_{j+1}, \varepsilon_{j+1}}^{I}\right)^{\prime}\left(N / B_{j+1}\right)\right) / b_{j+1}
\end{gathered}
$$

et

$$
N_{j} / b_{j+1} \leq N / B_{j+1}<\left(N_{j}+1\right) / b_{j+1} .
$$

Si $0 \leq \Lambda_{j} \leq \nu_{j}$, deux cas se présentent :

Ou bien $\Lambda_{j+1} \leq \nu_{j+1}$, donc $\lambda_{j+1} \leq N_{j}$ et, d'après l'hypothèse de récurrence, $\delta_{j+1}=\lambda_{j+1}=\varepsilon_{j+1}$; d'où $\left(\varphi_{b_{j+1}, \varepsilon_{j+1}}^{I}\right)^{\prime}\left(N / B_{j+1}\right)=-\varepsilon_{j+1}$ et $\delta_{j}=\lambda_{j}$.

Ou bien $\nu_{j+1}<\Lambda_{j+1}<B_{n} / B_{j+1}$, donc $\lambda_{j+1}<N_{j}$ et, par hypothèse de récurrence, $\delta_{j+1}=\lambda_{j+1}+1$; comme $N_{j}<b_{j+1}$, on a nécessairement $\lambda_{j+1}+1<b_{j+1}$; d'où $\varepsilon_{j+1}=\lambda_{j+1}+1,\left(\varphi_{b_{j+1}, \varepsilon_{j+1}}^{I}\right)^{\prime}\left(N / B_{j+1}\right)=-\varepsilon_{j+1}$ et $\delta_{j}=\lambda_{j}$.

Si $\nu_{j}<\Lambda_{j}<B_{n} / B_{j}$, en suivant le même plan on aboutit au résultat $\delta_{j}=\lambda_{j}+1$.

Dans le cas général où les permutations $\sigma_{j}$ sont quelconques, on se ramène au cas précédent : D'après la propriété 3.4 , on a

$$
\begin{aligned}
\left(\varphi_{b_{j+1}, \varepsilon_{j+1}}^{\sigma_{j}}\right)^{\prime}\left(N / B_{j+1}\right) & =\left(\varphi_{b_{j+1}, \varepsilon_{j+1}}^{\sigma_{j}}\right)^{\prime}\left(N_{j} / b_{j+1}\right) \\
& =\left(\varphi_{b_{j+1}, \varepsilon_{j+1}}^{I}\right)^{\prime}\left(\sigma_{j}\left(N_{j}\right) / b_{j+1}\right),
\end{aligned}
$$

d'où

$$
\delta_{j}=\lambda_{j}+\left(\delta_{j+1}+\left(\varphi_{b_{j+1}, \varepsilon_{j+1}}^{I}\right)^{\prime}\left(\sigma_{j}\left(N_{j}\right) / b_{j+1}\right)\right) / b_{j+1} ;
$$

on se trouve alors dans le cas des permutations identiques avec

$$
N^{\prime}=\sum_{h=0}^{n-1} \sigma_{h}\left(N_{h}\right) B_{h} \quad \text { et } \quad \nu_{j}^{\prime}=\sum_{h=j}^{n-1} \sigma_{h}\left(N_{h}\right) B_{n} / B_{h+1}=\nu_{j},
$$

d'où les relations sur les $\delta_{j}$.

Montrons maintenant les relations sur les $\varepsilon_{j}$ : Si $0 \leq \Lambda_{j-1} \leq \nu_{j}$, on a $\lambda_{j}=0$, d'où $\Lambda_{j-1}=\Lambda_{j}$ et donc $\delta_{j}=\varepsilon_{j}=0$. Si $1 \leq p<b_{j}$, ou bien $\Lambda_{j} \leq \nu_{j}$, d'où $\lambda_{j}=p$ donc $\delta_{j}=\lambda_{j}$ et par suite $\varepsilon_{j}=p$, ou bien $\nu_{j}<\Lambda_{j}$, d'où $\lambda_{j}=p-1$ donc $\delta_{j}=\lambda_{j}+1=p$ et par suite $\varepsilon_{j}=p$.

Dans le dernier cas, $\lambda_{j}=b_{j}-1$ et $\delta_{j}=\lambda_{j}+1$ car $\nu_{j}<\Lambda_{j}$, d'où $\delta_{j}=b_{j}$ et $\varepsilon_{j}=0$.

5.4. Démonstration du théorème 4.1. Pour alléger, nous omettrons ici d'écrire $S_{B}^{\Sigma}$. Nous devons calculer $T^{2}(N)=\int_{0}^{1} E^{2}(\alpha, N) d \alpha$. D'après 
le lemme 5.1 dont nous garderons les notations, dans chaque intervalle $\left[(\lambda-1) / B_{n}, \lambda / B_{n}\left[\right.\right.$, il y a un et un seul point de $\bar{X}_{n}$ noté $x_{\lambda}^{\prime}$ et, en outre,

$$
E(\alpha, N)= \begin{cases}E\left((\lambda-1) / B_{n}, N\right)+\left((\lambda-1) / B_{n}-\alpha\right) N & \text { si }(\lambda-1) / B_{n} \leq \alpha \leq x_{\lambda}^{\prime}, \\ E\left(\lambda / B_{n}, N\right)+\left(\lambda / B_{n}-\alpha\right) N \quad & \text { si } x_{\lambda}^{\prime}<\alpha<\lambda / B_{n} ;\end{cases}
$$

ces relations nous conduisent à intégrer sur $\left[(\lambda-1) / B_{n}, x_{\lambda}^{\prime}\right]$ et sur $\left[x_{\lambda}^{\prime}, \lambda / B_{n}\right]$. Le calcul fournit

$$
\begin{aligned}
T^{2}(N)= & \frac{1}{B_{n}} \sum_{\lambda=1}^{B_{n}} E^{2}\left(\frac{\lambda}{B_{n}}, N\right)-\frac{N}{B_{n}} \sum_{\lambda=1}^{B_{n}} E\left(\frac{\lambda}{B_{n}}, N\right)\left(x_{\lambda}^{\prime}+x_{\lambda+1}^{\prime}-2 \frac{\lambda}{B_{n}}\right) \\
& +\frac{N^{2}}{3 B_{n}} \sum_{\lambda=1}^{B_{n}-1}\left(\left(\frac{\lambda}{B_{n}}-x_{\lambda}^{\prime}\right)^{2}+\left(\frac{\lambda}{B_{n}}-x_{\lambda}^{\prime}\right)\left(\frac{\lambda}{B_{n}}-x_{\lambda+1}^{\prime}\right)\right. \\
& \left.+\left(\frac{\lambda}{B_{n}}-x_{\lambda+1}^{\prime}\right)^{2}\right)+\frac{N^{2}}{3}\left(x_{1}^{\prime 3}+\left(1-x_{B_{n}}^{\prime}\right)^{3}\right),
\end{aligned}
$$

formule qui se réduit à

$$
\begin{aligned}
T^{2}(N)= & \frac{1}{B_{n}} \sum_{\lambda=1}^{B_{n}} E^{2}\left(\lambda / B_{n}, N\right)-N B_{n}^{-1}\left(2 x_{1}-B_{n}^{-1}\right) \sum_{\lambda=1}^{B_{n}} E\left(\lambda / B_{n}, N\right) \\
& +N^{2}\left(3 x_{1}^{\prime 2}-3 x_{1}^{\prime} B_{n}^{-1}+B_{n}^{-2}\right) / 3
\end{aligned}
$$

car $x_{\lambda}^{\prime}-\lambda / B_{n}$ ne dépend pas de $\lambda$, d'après la propriété 3.2.

Calcul de $A=\sum_{\lambda=1}^{B_{n}} E\left(\lambda / B_{n}, N\right)$ : D'après le lemme 5.2,

$$
A=\sum_{j=1}^{n} \sum_{\lambda=1}^{B_{n}} \varphi_{b_{j}, \varepsilon_{j}}^{\sigma_{j-1}}\left(N / B_{j}\right) \quad \text { où les } \varepsilon_{j} \text { sont fonctions de } \lambda, n \text { et } N \text {. }
$$

Pour $j$ fixé, en remarquant que $\lambda=\lambda_{1} B_{n} / B_{1}+\ldots+\lambda_{j-1} B_{n} / B_{j-1}+\Lambda_{j-1}$, on voit que l'ensemble des $\lambda$ est partagé en $B_{n} / B_{j-1}$ classes de $B_{j-1}$ termes chacune où $\Lambda_{j-1}$ est constant; d'où, d'après le lemme 5.3 , quel que soit $p$ $\left(0 \leq p \leq b_{j}-1\right), \varepsilon_{j}(\lambda)=p$ pour $\left(B_{n} / B_{j}\right) B_{j-1}=B_{n} / b_{j}$ valeurs de $\lambda$. On en déduit

$$
A=\sum_{j=1}^{n}\left(\left(B_{n} / b_{j}\right) \sum_{p=0}^{b_{j}-1} \varphi_{b_{j}, p}^{\sigma_{j-1}}\left(N / B_{j}\right)\right)=B_{n} \sum_{j=1}^{n} \varphi_{b_{j}}^{\sigma_{j-1}}\left(N / B_{j}\right) / b_{j} .
$$

Calcul de $B=\sum_{\lambda=1}^{B_{n}} E^{2}\left(\lambda / B_{n}, N\right)$ : Toujours d'après le lemme 5.2,

$$
B=\sum_{j=1}^{n} \sum_{\lambda=1}^{B_{n}}\left(\varphi_{b_{j}, \varepsilon_{j}}^{\sigma_{j-1}}\right)^{2}\left(N / B_{j}\right)+2 \sum_{1 \leq i<j \leq n} \sum_{\lambda=1}^{B_{n}} \varphi_{b_{i}, \varepsilon_{i}}^{\sigma_{i-1}}\left(N / B_{i}\right) \varphi_{b_{j}, \varepsilon_{j}}^{\sigma_{j-1}}\left(N / B_{j}\right) .
$$


De la même façon que pour le calcul de $A$, la première somme double vaut $B_{n} \sum_{j=1}^{n} \phi_{b_{j}}^{\sigma_{j-1}}\left(N / B_{j}\right) / b_{j}$.

Fixons $i$ et $j$; rappelons que $\varepsilon_{j}(\lambda)=p$ pour $B_{n} / B_{j}$ classes de l'ensemble des $\lambda$ où $\Lambda_{j-1}$ est constant; plaçons-nous dans une de ces classes; d'après le lemme $5.3, \varepsilon_{i}(\lambda)=q$ pour $\left(B_{n} / B_{i}\right) /\left(B_{n} / B_{j-1}\right)=B_{j-1} / B_{i}$ sous-classes de $B_{i-1}$ termes chacune où $\Lambda_{i-1}$ est constant; d'où, quels que soient $p$ et $q, \varepsilon_{j}(\lambda)=p$ et $\varepsilon_{q}(\lambda)=q$ pour $\left(B_{n} / B_{j}\right)\left(B_{j-1} / B_{i}\right) B_{i-1}=B_{n} /\left(b_{i} b_{j}\right)$ valeurs de $\lambda$. On en déduit que la seconde somme double de $B$ vaut

$$
B_{n} \sum_{1 \leq i<j \leq n} \varphi_{b_{i}}^{\sigma_{i-1}}\left(N / B_{i}\right) \varphi_{b_{j}}^{\sigma_{j-1}}\left(N / B_{j}\right) /\left(b_{i} b_{j}\right)
$$

ce qui fournit

$$
\begin{aligned}
B= & B_{n} \sum_{j=1}^{n} \phi_{b_{j}}^{\sigma_{j-1}}\left(N / B_{j}\right) / b_{j} \\
& +2 B_{n} \sum_{1 \leq i<j \leq n} \varphi_{b_{i}}^{\sigma_{i-1}}\left(N / B_{i}\right) \varphi_{b_{j}}^{\sigma_{j-1}}\left(N / B_{j}\right) /\left(b_{i} b_{j}\right) .
\end{aligned}
$$

Les résultats étant valables pour $1 \leq N \leq B_{n}$, on obtient alors la formule du théorème en faisant tendre $n$ vers l'infini; pour cela, il suffit de remarquer que $\left|\varphi_{b_{i}}^{\sigma_{i}}\right|<b_{i}^{2} / 4$ d'après la propriété 3.4 , que $\sum_{j=1}^{n} b_{j} / B_{n}$ tend vers zéro quand $n$ tend vers l'infini et que $x_{1}<1 / B_{n}$.

5.5. Démonstration des théorèmes 4.2 et 4.3. D'après la formule de Koksma (propriété 2.2 ) et la relation $\sum_{n=1}^{N}\left(1 / 2-x_{n}\right)=\int_{0}^{1} E(\alpha, N, X) d \alpha$, on obtient

$$
(2 \pi)^{-2} F^{2}\left(N, S_{B}^{\Sigma}\right)=T^{2}\left(N, S_{B}^{\Sigma}\right)-\left(\int_{0}^{1} E\left(\alpha, N, S_{B}^{\Sigma}\right) d \alpha\right)^{2} .
$$

Comme dans 5.4, on montre que

$$
\begin{aligned}
\int_{0}^{1} E(\alpha & \left., N, S_{B}^{\Sigma}\right) d \alpha \\
& =B_{n}^{-1} \sum_{\lambda=1}^{B_{n}} E\left(\lambda / B_{n}, N\right)+N\left(2 B_{n}\right)^{-1} \sum_{\lambda=1}^{B_{n}}\left((2 \lambda-1) / B_{n}-2 x_{\lambda}^{\prime}\right) ;
\end{aligned}
$$

en utilisant l'expression de $\sum_{\lambda=1}^{B_{n}} E\left(\lambda / B_{n}, N\right)$ obtenue précédemment et en introduisant la fonction $\chi=b \phi-\varphi^{2}$, on obtient le théorème 4.2.

Remarquons que pour tout $h, \varphi_{b, h}^{I} \geq 0$ puisque

$$
\varphi_{b, h}^{I}(x)= \begin{cases}(b-h) x & \text { si } x \in[0, h / b] \\ h(1-x) & \text { si } x \in[h / b, 1]\end{cases}
$$

Par suite, d'après le lemme $5.2, E\left(\lambda / B_{n}, N, S_{B}^{I}\right)=\sum_{j=1}^{n} \varphi_{b_{j}, \varepsilon_{j}}^{I}\left(N / B_{j}\right)$ est 
positif pour tout $\lambda$; on en déduit que $E\left(\alpha, N, S_{B}^{I}\right)>0$ pour tout $\alpha$, puisque

$$
E\left(\alpha, N, S_{B}^{I}\right)=E\left(\lambda / B_{n}, N, S_{B}^{I}\right)+\left(\lambda / B_{n}-\alpha\right) N
$$

où $\lambda$ est défini par $(\lambda-1) / B_{n}<\alpha<\lambda / B_{n}$. D'où le théorème 4.3 puisque $\int_{0}^{1} E\left(\alpha, N, S_{B}^{I}\right) d \alpha=\sum_{j=1}^{\infty} \varphi_{b_{j}}^{I}\left(N / B_{j}\right) / b_{j}$.

5.6. Démonstration du théorème 4.4. Rappelons que

$$
\begin{gathered}
D\left(N, S_{b}^{\sigma}\right)=\sum_{j=1}^{\infty} \psi_{b}^{\sigma}\left(N / b^{j}\right) \quad[6], \quad F^{2}\left(N, S_{b}^{\sigma}\right)=4 \pi^{2} \sum_{j=1}^{\infty} \chi_{b}^{\sigma}\left(N / b^{j}\right) / b^{2}, \\
\psi_{b}^{\sigma}=\sup _{0 \leq h<h^{\prime}<b}\left|\varphi_{b, h}^{\sigma}-\varphi_{b, h^{\prime}}^{\sigma}\right| \quad \text { et } \quad \chi_{b}^{\sigma}=\frac{1}{2} \sum_{0 \leq h<h^{\prime}<b}\left(\varphi_{b, h}^{\sigma}-\varphi_{b, h^{\prime}}^{\sigma}\right)^{2} .
\end{gathered}
$$

Pour démontrer les propriétés, il suffit donc de montrer que l'ensemble des différences $\varphi_{h}-\varphi_{h^{\prime}}$ est le même pour les permutations $\sigma$ et $\tau$; pour cela, $\mathrm{du}$ fait que la translation de vecteur $(l, l)$ est une bijection de $\{0, \ldots, b-1\}^{2}$ sur lui-même, il suffit de montrer que $\varphi_{b, h}^{\sigma}-\varphi_{b, h^{\prime}}^{\sigma}=\varphi_{b, h_{1}}^{\tau}-\varphi_{b, h_{1}^{\prime}}^{\tau}$ où $h_{1}=$ $h+l(\bmod b)$ et $h_{1}^{\prime}=h^{\prime}+l(\bmod b)$. Les fonctions $\varphi_{b, h}^{\sigma}$ étant affines sur $[(k-1) / b, k / b]$ et continues, on peut se contenter de vérifier l'égalité en $k / b(1 \leq k \leq b)$. Or $\varphi_{b, h}^{\sigma}(k / b)=E\left(h / b, k, \mathcal{Z}_{b}^{\sigma}\right)$ d'après la définition 3.3 et $E\left(\left[h^{\prime} / b, h / b\left[; k ; \mathcal{Z}_{b}^{\sigma}\right)=E\left(\left[h_{1}^{\prime} / b, h_{1} / b\left[; k ; \mathcal{Z}_{b}^{\tau}\right)\right.\right.\right.\right.$, d'où le résultat.

Remarquons que les $b$ premiers termes de $S_{b}^{\sigma}$ et $S_{b}^{\tau}$ se correspondent dans la translation $(\tau(0)-\sigma(0)) /(b(b-1))+l / b(\bmod 1)$ et que cette propriété n'est plus vraie au-delà, d'où la remarque suivant le théorème 4.4.

\section{Démonstration des résultats du paragraphe $\mathbf{4 . 2}$}

6.1. Démonstration des théorèmes 4.5 et 4.6. D'après la propriété 2.1 , il suffit de démontrer le théorème 4.5 pour $D$. Rappelons que

$$
D\left(N, S_{B}^{\Sigma}\right)=\sum_{j=1}^{\infty} \psi_{b_{j}}^{\sigma_{j-1}}\left(N / B_{j}\right),
$$

$0 \leq \psi_{b_{j}}^{\sigma_{j-1}} \leq b_{j} / 4$ et $\psi_{b_{j}}^{\sigma_{j-1}}(x)=\left(b_{j}-1\right) x$ sur $\left[0,1 / b_{j}\right]$ (voir $\left.[6]\right)$.

Soient $N$ et $n$ tels que $B_{n-1} \leq N<B_{n}$; d'une part,

$$
\sum_{j=1}^{n} \psi_{b_{j}}^{\sigma_{j-1}}\left(N / B_{j}\right) \leq K(\log N / \log b+1) / 4
$$

$\operatorname{car}(n-1) \log b \leq \sum_{j=1}^{n-1} \log b_{j} \leq \log N$; d'autre part,

$$
\sum_{j=n+1}^{\infty} \psi_{b_{j}}^{\sigma_{j-1}}\left(N / B_{j}\right)=\sum_{j=n+1}^{\infty}\left(b_{j}-1\right) N / B_{j}=N / B_{n}<1
$$

car ici $N / B_{j}<1 / b_{j}$; le théorème 4.5 est alors immédiat. 
Nous majorons $\chi_{b_{j}}^{\sigma_{j-1}}$ par $b_{j} \phi_{b_{j}}^{\sigma_{j-1}}, \phi_{b_{j}}^{\sigma_{j-1}}$ vérifiant $\phi_{b_{j}}^{\sigma_{j-1}} \leq b_{j}^{2}\left(b_{j}-1\right) / 16$, et nous utilisons la propriété 3.5(ii) donnant $\chi_{b_{j}}^{\sigma}(x)$ sur $\left[0, b_{j}\right]$. D'une part,

$$
\sum_{j=1}^{n} \phi_{b_{j}}^{\sigma_{j-1}}\left(N / B_{j}\right) / b_{j} \leq(K-b) n / 16 ;
$$

d'autre part,

$$
\begin{aligned}
\sum_{j=n+1}^{\infty} \phi_{b_{j}}^{\sigma_{j-1}}\left(N / B_{j}\right) / b_{j}^{2} & =\left(N^{2} / 12\right) \sum_{j=n+1}^{\infty}\left(b_{j}^{2}-1\right) / B_{j}^{2} \\
& =N^{2} /\left(12 B_{n}^{2}\right)<1 / 12 ;
\end{aligned}
$$

d'où le théorème 4.6, car $B_{n-1} \leq N<B_{n}$ et $(n-1) \log b \leq \log N$.

6.2. Démonstration des théorèmes 4.7 et 4.8. Pour le théorème 4.7 , il suffit de démontrer que $\sum_{j=1}^{n} b_{j} \in O(n)$ si $D^{(1)}\left(N, S_{B}^{I}\right) \in$ $O(\log N)$, compte tenu du théorème 4.5 et de la propriété 2.1 .

D'après le théorème 4.3 , pour $B_{n-1} \leq N<B_{n}$,

$$
D^{(1)}\left(N, S_{B}^{I}\right)=\sum_{j=1}^{\infty} \varphi_{b_{j}}^{I}\left(N / B_{j}\right) / b_{j}=\sum_{j=1}^{n} \varphi_{b_{j}}^{I}\left(N / B_{j}\right) / b_{j}+N /\left(2 B_{n}\right)
$$

$\operatorname{car} \varphi_{b}^{I}(x)=b(b-1) x / 2$ sur $[0,1 / b]$ (voir la propriété 7.1 ); d'après cette même propriété, $\varphi_{b}^{I}$ atteint son maximum en $1 / 2$; de plus, $\varphi_{b}^{I}(x) \geq\left(b^{2}-4\right) / 8$ sur $[1 / 2,1 / 2+1 / b]$ si $b$ est pair et sur $[(b-1) /(2 b),(b+1) /(2 b)]$ si $b$ est impair.

Considérons la suite

$$
N_{n}=\sum_{i=0}^{n-1}\left[b_{i+1} / 2\right] B_{i}
$$

(où $[x]$ désigne la partie entière de $x$ ); on vérifie aisément que $1 / 2 \leq\left\{N_{n} / B_{j}\right\}$ $<1 / 2+1 / b_{j}$ si $b_{j}$ est pair et $\left(b_{j}-1\right) /\left(2 b_{j}\right) \leq\left\{N_{n} / B_{j}\right\}<\left(b_{j}+1\right) /\left(2 b_{j}\right)$ si $b_{j}$ est impair (où $\{x\}$ est la partie fractionnaire de $x$ ); on obtient alors

$$
\sum_{j=1}^{n} \varphi_{b_{j}}^{I}\left(N_{n} / B_{j}\right) / b_{j} \geq \sum_{j=1}^{n}\left(b_{j}^{2}-4\right) /\left(8 b_{j}\right) \geq \sum_{j=1}^{n} b_{j} / 8-n /(2 b)
$$

où $b=\inf b_{j}$. Par hypothèse $D^{(1)}\left(N, S_{B}^{I}\right) \leq K \log N$, d'où

$$
\sum_{j=1}^{n} b_{j} / 8-n /(2 b) \leq K \log N_{n}<K \log B_{n}=K \sum_{j=1}^{n} \log b_{j} .
$$

Par convexité de la fonction Log, en posant $M_{n}=\sum_{j=1}^{n} b_{j} / n$, on obtient

$$
M_{n} \leq 4 / b+8 K \log M_{n} ;
$$

on en déduit que $M_{n}$ est bornée, c'est-à-dire $\sum_{i=1}^{n} b_{i} \in O(n)$. 
Remarquons qu'un calcul plus précis donne, pour $K \geq 1, \sum_{j=1}^{n} b_{j} \leq C_{n}$ avec $C=4+16 K \log (8 K \log (16 K \log (8 K)))-16 K$.

Grâce à la propriété 7.1(iii), la majoration du théorème 4.6 peut être améliorée si $\Sigma=I$; on obtient

$$
F^{2}\left(N, S_{B}^{I}\right) \leq(C / \log b) \log N+C+\pi^{2} / 3 \quad \text { avec } C=(K+8) \pi^{2} / 48,
$$

d'où la condition suffisante du théorème 4.8 .

Réciproquement, avec la suite $\left(N_{n}\right)$ utilisée précédemment, nous obtenons

$$
\chi_{b_{j}}^{I}\left(N_{n} / B_{j}\right) \geq\left(b_{j}^{4}-b_{j}^{2}-9 b_{j}^{2} /\left(b_{j}^{2}-1\right)\right) / 192,
$$

en utilisant la minoration de $\chi_{b}^{I}$ sur $[1 / 2,(b+2) /(2 b)]$ si $b$ est pair et sur $[(b-1) /(2 b),(b+1) /(2 b)]$ si $b$ est impair (voir propriété $7.1(i i i)$ ); en ajoutant ces inégalités on aboutit, toujours avec $b=\inf b_{j}$, à

$$
\sum_{j=1}^{n} \chi_{b_{j}}^{I}\left(N_{n} / B_{j}\right) / b_{j}^{2} \geq\left(\pi^{2} / 48\right)\left(\sum_{j=1}^{n} b_{j}^{2}-\left(1+9 /\left(b^{2}-1\right)\right) n\right) .
$$

Par hypothèse $F^{2}\left(N_{n}, S_{B}^{I}\right) \leq K \log N_{n}$ et, comme $N_{n}<B_{n}$, on a

$$
K \sum_{j=1}^{n} \log b_{j} \geq\left(\pi^{2} / 48\right)\left(\sum_{j=1}^{n} b_{j}^{2}-\left(1+9 /\left(b^{2}-1\right)\right) n\right) ;
$$

en posant $M_{n}=\sum_{j=1}^{n} b_{j}^{2} / n$, par convexité du logarithme, il en résulte que

$$
M_{n} \leq 1+9 /\left(b^{2}-1\right)+\left(24 K / \pi^{2}\right) \log M_{n} .
$$

On en déduit encore que $M_{n}$ est bornée, c'est-à-dire $\sum_{j=1}^{n} b_{j}^{2} \in O(n)$.

Ici aussi, un calcul plus détaillé donne une constante du même type que précédemment pour le théorème 4.7 .

\subsection{Démonstration des théorèmes 4.9 et 4.10}

6.3.1. Lemme. Soit $f$ une fonction réelle bornée; on pose

$$
d_{n}=\sup _{x \in \mathbb{R}}\left|\sum_{j=1}^{n} f\left(x / b^{j}\right)\right| \quad \text { et } \quad \alpha=\inf _{n \geq 1} d_{n} / n
$$

alors on a

$$
\alpha=\lim _{n \rightarrow+\infty} d_{n} / n .
$$

Preuve. Pour $n$ et $m$ donnés, soient $q$ et $r$ le quotient et le reste de la division de $m$ par $n$. En décomposant $\sum_{j=1}^{m} f\left(x / b^{j}\right)$ en $q+1$ sommes, on obtient

$$
d_{m} \leq q d_{n}+d_{r} \quad \text { car } \quad\left|\sum_{j=\nu n+1}^{\nu(n+1)} f\left(x / b^{j}\right)\right|=\left|\sum_{h=1}^{n} f\left(\left(x / b^{\nu n}\right) / b^{h}\right)\right| \leq d_{n} .
$$


Il en résulte que $d_{m} / m \leq d_{n} / n-(r / m)\left(d_{n} / n\right)+d_{r} / m$ et, en faisant tendre $m$ vers l'infini,

$$
\limsup _{m \rightarrow+\infty} d_{m} / m \leq d_{n} / n \quad \text { pour tout } n,
$$

d'où la propriété annoncée.

Rem arque. Si $f$ est de période 1 , en posant $g_{n}(x)=\sum_{k=0}^{n-1} f\left(x b^{k}\right)$, on a aussi $d_{n}=\sup _{x \in[0,1]}\left|g_{n}(x)\right|$.

6.3.2. Lemme. Soit $f$ une fonction réelle bornée de période 1; avec $g_{n}(x)=\sum_{k=0}^{n-1} f\left(x b^{k}\right)$, on a $\left|g_{\nu}\left(a /\left(b^{\nu}-1\right)\right)\right| \leq \nu \alpha$ quels que soient les entiers $a$ et $\nu$ tels que $1 \leq a<b^{\nu}$, $\alpha$ étant défini comme au lemme 6.3.1.

Preuve. En remarquant que

$$
\sum_{k=h \nu}^{(h+1) \nu-1} f\left(a b^{k} /\left(b^{\nu}-1\right)\right)=\sum_{l=0}^{\nu-1} f\left(a b^{l} /\left(b^{\nu}-1\right)\right)
$$

puisque $a b^{\nu h}=a \bmod \left(b^{\nu}-1\right)$ et que $f$ est 1-périodique, on obtient

$$
g_{\nu n}\left(a /\left(b^{\nu}-1\right)\right)=n g_{\nu}\left(a /\left(b^{\nu}-1\right)\right) .
$$

On en déduit que

$$
\left|g_{\nu}\left(a /\left(b^{\nu}-1\right)\right)\right| / \nu \leq d_{\nu n} /(\nu n)
$$

d'où $\left|g_{\nu}\left(a /\left(b^{\nu}-1\right)\right)\right| / \nu \leq \alpha$ car pour $\nu$ fixé $d_{\nu n} /(\nu n)$ est suite extraite de $d_{m} / m$ qui admet $\alpha$ pour limite d'après le lemme 6.3.1.

6.3.3. Lemme. Si la fonction $f$ du lemme 6.3 .1 est $\varphi_{b}^{\sigma}$, alors $\alpha=\beta_{b}^{\sigma}$ est strictement positif.

Preuve. Cas $b=2: \psi_{2}^{I}=\varphi_{2}^{I}=-\varphi_{2}^{J}$ avec $J=(10)$; par suite, $\left|g_{2}(a / 3)\right|=2 / 3$ pour $a=1$ ou 2 , d'où $\alpha \geq 1 / 3$ (en fait $\alpha=1 / 3$ d'après [7], notamment).

Cas $b \geq 3$ : Rappelons (voir propriété 3.5) que

$$
\varphi_{b}^{\sigma}(x)=b((b-1) / 2-\sigma(1))(x-1 / b)+(b-1) / 2-\sigma(0) \quad \text { sur }[1 / b, 2 / b],
$$

d'où $\varphi_{b}^{\sigma}(1 /(b-1))=b / 2-\sigma(1) /(b-1)-\sigma(0)$.

Si $b$ est impair, $\varphi_{b}^{\sigma}(1 /(b-1))$ ne peut être nul que si $\sigma(1) /(b-1)=$ $1 / 2$ puisque $0 \leq \sigma(k) \leq b-1$; mais alors on aboutit à la contradiction $\sigma(0)=\sigma(1)$. On en déduit donc que $g_{1}(1 /(b-1)) \neq 0$ et $\alpha>0$ d'après le lemme 6.3.2.

Si $b$ est pair, $\varphi_{b}^{\sigma}(1 /(b-1))$ ne peut être nul que si $\sigma(1)=0$ ou $b-1$.

Dans le cas $\sigma(1)=0, \varphi_{b}^{\sigma}(1 /(b-1))$ est nul si de plus $\sigma(0)=b / 2$; il suffit alors de calculer $\varphi_{b}^{\sigma}(2 /(b-1))=b / 2-2 \sigma(2) /(b-1)$, d'après la propriété 3.5 ; il en résulte que $\varphi_{b}^{\sigma}(2 /(b-1))$ ne peut être nul que si $b=4$; reste donc à regarder le cas particulier $b=4$ et $\sigma=\left(\begin{array}{llll}2 & 0 & 3 & 1\end{array}\right)$ pour lequel $g_{2}(1 / 15)=-8 / 15$; on a donc bien $\alpha>0$ dans le cas $\sigma(1)=0$. Le cas 
$\sigma(1)=b-1$ se traite de manière analogue : $g_{1}(1 /(b-1))$ ne peut être nul que si $\sigma(0)=b / 2-1$; alors $\varphi_{b}^{\sigma}(2 /(b-1))=(4-b) / 2-\sigma(2) /(b-1)$ ne peut

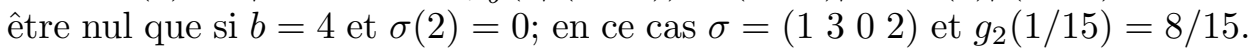

En conclusion, on obtient

$$
\alpha \geq\left|g_{1}(1 /(b-1))\right| \text { ou } \alpha \geq\left|g_{1}(2 /(b-1))\right| \text { ou } 2 \alpha \geq\left|g_{2}\left(1 /\left(b^{2}-1\right)\right)\right|,
$$

donc $\alpha>0$ dans tous les cas.

6.3.4. Démonstration du théorème 4.9. Grâce à la remarque 3 suivant le théorème 4.6 , en base fixe, on a

$$
T^{2}\left(N, S_{b}^{\sigma}\right)=\left(\sum_{j=1}^{\infty} \varphi_{b}^{\sigma}\left(N / b^{j}\right) / b\right)^{2}+O(\log N) .
$$

Soient $N$ et $n$ tels que $b^{n} \leq N<b^{n+1}$; alors

$$
\left|\sum_{j=n+1}^{\infty} \varphi_{b}^{\sigma}\left(N / b^{j}\right)\right| \leq b^{2} / 4+\left|\sum_{j=n+2}^{\infty} \varphi_{b}^{\sigma}\left(N / b^{j}\right)\right| \leq b^{2} / 4+b / 2,
$$

en utilisant l'expression de $\varphi_{b}^{\sigma}$ sur $[0,1 / b]$; d'où

$$
T^{2}\left(N, S_{b}^{\sigma}\right)=\left(\sum_{j=1}^{n} \varphi_{b}^{a}\left(N / b^{j}\right) / b\right)^{2}+O(\log N) \quad \text { puisque } \quad\left|\varphi_{b}^{\sigma}\right|<b^{2} / 4 .
$$

Montrons alors que

$$
\limsup _{N \rightarrow+\infty}\left|\sum_{j=1}^{n} \varphi_{b}^{\sigma}\left(N / b^{j}\right) / \log N\right|=\beta_{b}^{\sigma} / \log b .
$$

D'après le lemme 6.3.1, appliqué à $f=\varphi_{b}^{\sigma}$, on a

$$
\left|\sum_{j=1}^{n} \varphi_{b}^{\sigma}\left(N / b^{j}\right)\right| / \log N \leq d_{n} / \log N
$$

d'où $\beta_{b}^{\sigma} / \log b$ majore la limite supérieure étudiée.

La fonction $\left|\sum_{j=1}^{n} \varphi_{b}^{\sigma}\left(x / b^{j}\right)\right|$ est continue et $b^{n}$-périodique, donc elle atteint son maximum $d_{n}$ en un point $N_{n}$ de $\left[b^{n}, 2 b^{n}\right.$ [; de plus, $N_{n}$ est entier car $\varphi_{b}^{\sigma}$ est affine sur les intervalles $[(k-1) / b, k / b]$. On a donc une suite d'entiers $\left(N_{n}\right)$ strictement croissante telle que

$$
d_{n} /((n+1) \log b) \leq \sum_{j=1}^{n} \varphi_{b}^{\sigma}\left(N_{n} / b^{j}\right) / \log N_{n} \leq d_{n} /(n \log b),
$$

d'où

$$
\lim _{n \rightarrow+\infty} \sum_{j=1}^{n} \varphi_{b}^{\sigma}\left(N_{n} / b^{j}\right) / \log N_{n}=\beta_{b}^{\sigma} / \log b
$$


ce qui achève la démonstration car, d'après le lemme $6.3 .3, \beta_{b}^{\sigma}$ n'est pas nul.

6.3.5. Démonstration du théorème 4.10. Grâce au théorème 4.2 , pour $b^{n} \leq N<b^{n+1}$,

$$
F^{2}\left(N, S_{b}^{\sigma}\right)=4 \pi^{2} \sum_{j=1}^{n} \chi_{b}^{\sigma}\left(N / b^{j}\right) / b^{2}+O(1) .
$$

De la même manière que dans 6.3.4, on a

$$
\limsup _{N \rightarrow+\infty} \sum_{j=1}^{n} \chi_{b}^{\sigma}\left(N / b^{j}\right) / \log N \leq \gamma_{b}^{\sigma} / \log b .
$$

La fonction $\chi_{b}^{\sigma}$ atteint son maximum en un point $k / b$ (voir propriété 3.5); d'où le maximum de la fonction $b^{n}$-périodique $\sum_{j=1}^{n} \chi_{b}^{\sigma}\left(x / b^{j}\right)$ est atteint pour un entier $N_{n}$ de $\left[b^{n}, 2 b^{n}\right.$ [ et on conclut de la même façon que dans 6.3.4 avec le lemme 6.3.1.

\section{Démonstration des résultats du paragraphe $\mathbf{4 . 3}$}

\subsection{Propriétés des fonctions $\varphi_{b}^{I}$ et $\chi_{b}^{I}$}

(i) Pour $0 \leq k \leq b-1$, sur $[k / b,(k+1) / b]$,

$$
\varphi_{b}^{I}(x)=(b / 2)(b-2 k-1)(x-k / b)+k(b-k) / 2 ;
$$

(ii) $\varphi_{b}^{I}$ est symétrique par rapport à la droite $x=1 / 2$, elle est maximum en $1 / 2$ et

- si b est impair

$$
\varphi_{b}^{I}(x)=\left(b^{2}-1\right) / 8 \quad \text { sur }[1 / 2-1 /(2 b), 1 / 2+1 /(2 b)],
$$

- si b est pair

$$
\left(b^{2}-4\right) / 8 \leq \varphi_{b}^{I}(x) \leq \varphi_{b}^{I}(1 / 2)=b^{2} / 8 \quad \text { sur }[1 / 2-1 / b, 1 / 2+1 / b] ;
$$

(iii) pour $0 \leq k \leq b-1$,

$$
\chi_{b}^{I}(k / b)=k(b-k)(2+k(b-k)) / 12 ;
$$

(iv) $\chi_{b}^{I}$ est symétrique par rapport à la droite $x=1 / 2$;

- si b est impair, sur $[1 / 2-1 /(2 b), 1 / 2+1 /(2 b)]$,

$$
\chi_{b}^{I}(x)=b^{2}\left(b^{2}-1\right)(x-1 / 2)^{2} / 12+\chi_{b}^{I}(1 / 2)
$$

avec $\chi_{b}^{I}(1 / 2)=\left(b^{2}-1\right)\left(b^{2}+3\right) / 192 ;$

- si b est pair, sur $[1 / 2-1 / b, 1 / 2]$,

$$
\chi_{b}^{I}(x)=b^{2}\left(b^{2}-1\right)(x-1 / 2)^{2} / 12+b^{3}(x-1 / 2) / 8+\chi_{b}^{I}(1 / 2)
$$


et, sur $[1 / 2,1 / 2+1 / b]$,

$$
\chi_{b}^{I}(x)=b^{2}\left(b^{2}-1\right)(x-1 / 2)^{2} / 12-b^{3}(x-1 / 2) / 8+\chi_{b}^{I}(1 / 2)
$$

avec $\chi_{b}^{I}(1 / 2)=b^{2}\left(b^{2}+8\right) / 192$.

Preuve. Les propriétés (i) et (ii) résultent de la continuité des fonctions $\varphi$ et de la propriété 3.5 appliquées à la permutation identique.

Pour les propriétés (iii) et (iv), rappelons que

$$
\chi_{b}^{I}=b \sum_{h=0}^{b-1}\left(\varphi_{b, h}^{I}\right)^{2}-\left(\varphi_{b}^{I}\right)^{2}
$$

et que

il en résulte

$$
\varphi_{b, h}^{I}(x)= \begin{cases}(b-h) x & \text { si } x \in[0, h / b] \\ h(1-x) & \text { si } x \in[h / b, 1]\end{cases}
$$

$$
\chi_{b}^{I}(k / b)=\left(\sum_{j=1}^{k} j^{2}(b-k)^{2}+\sum_{j=1}^{b-k-1} k^{2} j^{2}\right) / b-k^{2}(b-k)^{2} / 4,
$$

ce qui donne, après calcul, la propriété (iii).

D'après la propriété 3.5(ii) et la formule précédente, la fonction $\chi_{b}^{I}$ est symétrique par rapport à $x=1 / 2$, puisque $\chi_{b}^{I}((b-k) / b)=\chi_{b}^{I}(k / b)$ et que sur chaque intervalle $[(k-1) / b, k / b]$ le coefficient en $x^{2}$ de $\chi_{b}^{I}$ est constant et vaut $b^{2}\left(b^{2}-1\right) / 12$. La connaissance de $\chi_{b}^{I}(k / b)$ permet aussi d'obtenir les équations de $\chi_{b}^{I}$ sur ces intervalles; nous les explicitons sur les seuls intervalles qui seront utiles par la suite (intervalles contenant $1 / 2$ ).

7.2. Démonstration du théorème 4.12. D'après la propriété 2.1 , le théorème 4.3 et le théorème 4.9 , il suffit de calculer

$$
\beta_{b}^{I}=\inf _{n \geq 1} \sup _{x \in \mathbb{R}}\left(\sum_{j=1}^{n} \varphi_{b}^{I}\left(x / b^{j}\right) / n\right)
$$

puisque $\varphi_{b}^{I} \geq 0$. D'après le lemme 6.3.1,

$$
\beta_{b}^{I}=\lim _{n \rightarrow+\infty} d_{n} / n \quad \text { où } d_{n}=\sup _{x \in[0,1]} g_{n}(x)=\sup _{x \in[0,1]} \sum_{k=0}^{n-1} \varphi_{b}^{I}\left(x b^{k}\right) .
$$

Pour calculer $d_{n}$, distinguons les cas $b$ pair et $b$ impair.

7.2.1. Cas b impair. D'après la propriété 7.1, $\varphi_{b}^{I}$ est croissante sur $[0,1 / 2-1 /(2 b)]$, constante sur $[1 / 2-1 /(2 b), 1 / 2+1 /(2 b)]$ où elle vaut $\left(b^{2}-1\right) / 8$ et décroissante sur $[1 / 2+1 /(2 b), 1]$; donc

$$
\sup _{x \in[0,1]}\left(\sum_{k=1}^{n-1} \varphi_{b}^{I}\left(x b^{k}\right)\right)=n\left(b^{2}-1\right) / 8,
$$


d'où le résultat puisque

$$
d^{(1)}\left(S_{b}^{I}\right)=d^{(2)}\left(S_{b}^{I}\right)=t_{1}\left(S_{b}^{I}\right)=\left(b^{2}-1\right) /(8 b \log b) .
$$

7.2.2. Cas b pair. Ici, $\varphi_{b}^{I}$ est croissante sur $[0,1 / 2]$ et symétrique par rapport à $x=1 / 2$; le calcul de $d_{n}$ est plus compliqué que dans le cas précédent car nous n'avons plus d'intervalle où $\varphi_{b}^{I}$ est constante et maximum. La démonstration se fait par la récurrence suivante suggérée par les premières itérations.

Hypothèse de récurrence. En posant $u_{n}=b\left(1-(-b)^{-n}\right) /(2(b+$ 1)) et $I_{n}=\left[u_{n}, u_{n+1}\right]$ si $n$ est pair, $I_{n}=\left[u_{n+1}, u_{n}\right]$ si $n$ est impair, on a

$$
d_{n}=g_{n}\left(u_{n}\right)= \begin{cases}\frac{b^{2}}{8} & \text { si } n=1, \\ n \frac{b^{3}+b^{2}-2 b}{8(b+1)}-\frac{b}{4(b+1)^{2}}\left(1-\left(\frac{-1}{b}\right)^{n-1}\right) & \text { si } n \geq 2 ;\end{cases}
$$

sur $I_{n}, g_{n}$ a pour pente $p_{n}=b\left(1-(-b)^{n}\right) /(2(b+1))$; de plus, les pentes des fonctions affines constituant $g_{n}$ sont en valeur absolue minorées par $\left|p_{n}\right|$.

D'après la propriété 7.1 , l'hypothèse est vraie pour $n=1$. Pour passer de $n$ à $n+1$, d'après l'hypothèse, il suffit d'étudier $g_{n+1}(x)=g_{n}(x)+\varphi_{b}^{I}\left(x b^{n}\right)$ sur $I_{n}$; en comparant les différentes valeurs de

$$
g_{n+1}\left(u_{n}+(-1)^{n} k / b^{n+1}\right)=g_{n}\left(u_{n}\right)+(-1)^{n} k p_{n} / b^{n+1}+\varphi\left((-1)^{n} k / b\right),
$$

on constate que la propriété au rang $n+1$ est vraie car on obtient $u_{n+1}=$ $u_{n}+(-b)^{-n} / 2, p_{n+1}=p_{n}-(-b)^{n} / 2$ et $d_{n+1}=d_{n}+p_{n}(-b)^{-n} / 2+b^{2} / 8$.

7.3. Démonstration du théorème 4.13. D'après le théorème 4.10 et le lemme 6.3.1, il s'agit de calculer

$$
\gamma_{b}^{I}=\inf _{n \geq 1} \sup _{x \in \mathbb{R}}\left(\sum_{j=1}^{n} \chi_{b}^{I}\left(x / b^{j}\right) / n\right)
$$

c'est-à-dire

$$
\lim _{n \rightarrow+\infty} d_{n} / n \quad \text { où } d_{n}=\sup _{x \in[0,1]} g_{n}(x)=\sup _{x \in[0,1]} \sum_{k=0}^{n-1} \chi_{b}^{I}\left(x b^{k}\right) .
$$

Pour calculer $d_{n}$, nous sommes amenés à distinguer les cas $b$ impair et $b$ pair; dans les deux cas, on élabore l'hypothèse de récurrence en s'appuyant sur les propriétés obtenues pour les premières valeurs de $n$, puis on la démontre en utilisant la relation $g_{n+1}(x)=g_{n}(x)+\chi_{b}^{I}\left(x b^{n}\right)$. 
7.3.1. Cas b impair $(b=2 p+1)$

Hypothèse de récurrence. En posant $u_{n}=1 / 2-1 /\left(2 b^{n}\right)$ et $I_{n}=\left[u_{n}, 1 / 2\right]$, on $a$

$$
g_{n}(x)=b^{2}\left(b^{2 n}-1\right)(x-1 / 2)^{2} / 12+n \chi_{b}^{I}(1 / 2) \quad \text { sur } I_{n} ;
$$

en outre $g_{n}$ est dominée par sa restriction à $I_{n}$ à symétrie et translation près (du fait de la symétrie de la fonction $\chi_{b}^{I}$ par rapport à la droite $x=1 / 2$ ).

Remarquons que

$$
d_{n}=g_{n}\left(u_{n}\right)=n \chi_{b}^{I}(1 / 2)+\left(b^{2 n}-1\right) /\left(48 b^{2 n-2}\right),
$$

d'où $\lim _{n \rightarrow+\infty} d_{n} / n=\chi_{b}^{I}(1 / 2)$, ce qui fournit le résultat du théorème 4.13 en utilisant la propriété 7.1(iv) et le théorème 4.10.

Vérification de l'hypothèse pour $n=1$. D'après la propriété 3.5 (ii), sur chaque intervalle $[k / b,(k+1) / b]$ le coefficient en $x^{2}$ de la fonction $g_{1}=\chi_{b}^{I}$ est constant et vaut $b^{2}\left(b^{2}-1\right) / 12$; il suffit de démontrer que

$$
\chi_{b}^{I}((k+1) / b)>\chi_{b}^{I}(k / b) \quad \text { pour } 0 \leq k \leq p-1,
$$

car le graphe de $\chi_{b}^{I}$, qui est continue, est constitué d'arcs translatés de la même parabole (en effet, si deux arcs ont une extrémité commune, l'un domine l'autre à symétrie près).

D'après la propriété 7.1 , après calcul,

$$
\chi_{b}^{I}((k+1) / b)-\chi(k / b)=\left(4 k^{3}-6(b-1) k^{2}+2 b(b-3) k+b^{2}-1\right) / 12 ;
$$

l'étude de la fonction du second membre montre que

$$
\chi_{b}^{I}((k+1) / b)-\chi_{b}^{I}(k / b) \geq\left(b^{2}-1\right) / 12 \quad \text { pour } 0 \leq k \leq p-1,
$$

l'égalité ayant lieu pour $k=0$ et $p-1$.

L'hypothèse de récurrence est donc vraie pour $n=1$.

Passage du rang $n$ a u rang $n+1$. Dans l'intervalle $I_{n}$, considérons la subdivision $z_{k}=1 / 2-1 /\left(2 b^{n}\right)+k / b^{n+1}$ pour $0 \leq k \leq p$; on a

$$
g_{n+1}\left(z_{k}\right)=g_{n}\left(z_{k}\right)+\chi_{b}^{I}(k / b)
$$

car $z_{k} b^{n}=\left(b^{n}-1\right) / 2+k / b$ et $\left(b^{n}-1\right) / 2$ est entier.

Pour déterminer $I_{n+1}$, il suffit de montrer que $g_{n+1}\left(z_{k+1}\right)>g_{n+1}\left(z_{k}\right)$ pour $0 \leq k \leq p-1$ (pour les mêmes raisons que ci-dessus pour $n=1$ ). Or

$$
\begin{aligned}
& g_{n+1}\left(z_{k+1}\right)-g_{n+1}\left(z_{k}\right) \\
& \quad=\frac{b^{2 n}-1}{12 b^{2 n}}\left(\left(\frac{b}{2}-k-1\right)^{2}-\left(\frac{b}{2}-k\right)^{2}\right)+\chi_{b}^{I}\left(\frac{k+1}{b}\right)-\chi_{b}^{I}\left(\frac{k}{b}\right)
\end{aligned}
$$

et, d'après le calcul fait pour $n=1$,

$$
g_{n+1}\left(z_{k+1}\right)-g_{n+1}\left(z_{k}\right) \geq\left(b^{2}-b+2 k\right) / 12+(b-2 k-1) /\left(12 b^{2 n}\right),
$$


d'où la propriété annoncée. Sur $I_{n}$, la fonction $g_{n+1}$ est formée d'arcs translatés de la même parabole avec l'ordonnée $g_{n+1}\left(z_{k}\right)$ croissante avec $k$, ce qui fournit $I_{n+1}$ compte tenu de la symétrie par rapport à $x=1 / 2$ des fonctions $g_{n}$ et $\chi_{b}^{I}$.

L'expression de $g_{n+1}$ découle immédiatement de $g_{n+1}(x)=g_{n}(x)+$ $\chi_{b}^{I}\left(x b^{n}\right)$. Ceci achève la démonstration du cas impair.

7.3.2. Cas b pair $(b=2 p)$

Hypothèse de récurrence. En posant $u_{n}=b\left(1-(-b)^{-n}\right) /(2(b+$ 1)) et $I_{n}=\left[u_{n}, u_{n+1}\right]$ si $n$ est pair, $I_{n}=\left[u_{n+1}, u_{n}\right]$ si $n$ est impair, on a

$$
g_{n}(x)=b^{2}\left(b^{2 n}-1\right) x^{2} / 12+\lambda_{n} x+\mu_{n} \quad \text { sur } I_{n},
$$

où $\lambda_{n}=\left(-2 b^{2 n+1}+(-1)^{n+1} b^{n+1}+(-1)^{n+1} 2 b^{n}+3 b+2\right) b^{2} /(24(b+1))$ (notons que le calcul de $\mu_{n}$ n'est pas utile dans la suite); de plus, $g_{n}$ est dominée par sa restriction à $I_{n}$ à symétrie et translation près et

$$
\begin{aligned}
d_{n}=g_{n}\left(u_{n}\right)= & n b^{2}\left(b^{3} / 4+b^{2} / 4+1\right) /(48(b+1))+b^{3} /(24(b+1)) \\
& +(-1)^{n+1}(b+2) /\left(48(b+1)^{2} b^{n-3}\right)-1 /\left(48(b+1)^{2} b^{2 n-4}\right) .
\end{aligned}
$$

On déduit de l'hypothèse de récurrence $\lim _{n \rightarrow+\infty} d_{n} / n$ et le résultat du théorème 4.13 en découle dans le cas $b$ pair.

Vérification de l'hypothèse pour $n=1$. Comme pour le cas $b$ impair et pour les mêmes raisons, il suffit de montrer que

$$
\chi_{b}^{I}((k+1) / b)>\chi_{b}^{I}(k / b) \quad \text { pour } 0 \leq k \leq p-1 ;
$$

un calcul analogue au cas $b$ impair donne

$$
\chi_{b}^{I}((k+1) / b)-\chi_{b}^{I}(k / b) \geq\left(b^{2}+2\right) / 24,
$$

l'égalité ayant lieu uniquement pour $k=p-1$.

Passage du rang $n$ a u rang $n+1$. Dans l'intervalle $I_{n}$, considérons la subdivision $z_{k}=u_{n}+(-1)^{n} k / b^{n+1}$ pour $0 \leq k \leq p$; on a

$$
g_{n+1}\left(z_{k}\right)=g_{n}\left(z_{k}\right)+\chi_{b}^{I}(k / b) .
$$

Pour déterminer $I_{n+1}$, il suffit de montrer que $g_{n+1}\left(z_{k+1}\right)>g_{n+1}\left(z_{k}\right)$ pour $0 \leq k \leq p-1 ;$ or

$$
\begin{aligned}
g_{n+1}\left(z_{k+1}\right)-g_{n+1}\left(z_{k}\right)= & b^{2}\left(b^{2 n}-1\right)\left((-1)^{n} 2 u_{n} / b^{n+1}+(2 k+1) / b^{2 n+2}\right) / 12 \\
& +(-1)^{n} \lambda_{n} / b^{n+1}+\chi_{b}^{I}((k+1) / b)-\chi_{b}^{I}(k / b),
\end{aligned}
$$

c'est-à-dire, d'après la propriété 7.1(iii),

$g_{n+1}\left(z_{k+1}\right)-g_{n+1}\left(z_{k}\right)=k^{3} / 3-(b-1) k^{2} / 2+k\left(b^{2}-3 b+1-1 / b^{2 n}\right) / b+a$ où $a=\left(b\left(2 b^{2}-b-2\right)+(-1)^{n}(b+2) b^{1-n}+2\left(b^{2}-b-1\right) b^{-2 n}\right) /(24(b+1))$.

L'étude de la fonction en $k$ du second membre fait apparaître qu'elle est croissante puis décroissante sur [0,p-1]; pour conclure sur le signe de 
l'expression étudiée, il suffit de calculer les valeurs en 0 et $p-1$ qui sont respectivement $a$ et $a-(b-2)\left(b+2 b^{-2 n}\right) / 24$; en explicitant $a$, on obtient que cette dernière expression est supérieure à

$$
\left(b^{n+2}+(-1)^{n}(b+2)\right) /\left(24(b+1) b^{n-1}\right),
$$

qui est positive.

Comme pour le cas $b$ impair, les ordonnées $g_{n+1}\left(z_{k}\right)$ sont croissantes avec $k$, ce qui donne $I_{n+1}$.

Pour exprimer $g_{n+1}(x)$ sur $I_{n+1}$, il faut connaître $\chi_{b}^{I}\left(x b^{n}\right)$ sur $I_{n+1}$; en séparant les cas $n$ pair et $n$ impair et en utilisant les expressions de $\chi_{b}^{I}$ sur $[1 / 2-1 / b, 1 / 2+1 / b]$ fournies par la propriété 7.1 , on obtient

$$
\chi_{b}^{I}\left(x b^{n}\right)=b^{2 n+2}\left(b^{2}-1\right) x^{2} / 12+\lambda x+\mu
$$

où

$$
\begin{aligned}
\lambda=\left(-2 b^{2 n+5}+2 b^{2 n+3}+\right. & (-1)^{n} b^{n+4} \\
& \left.+(-1)^{n} 3 b^{n+3}+(-1)^{n} 2 b^{n+2}\right) /(24(b+1)) ;
\end{aligned}
$$

on vérifie alors que $\lambda_{n+1}=\lambda_{n}+\lambda$, ce qui montre que l'hypothèse sur $\lambda_{n}$ est vraie au rang $n+1$.

Pour achever la démonstration il reste à montrer que l'hypothèse faite sur $d_{n}$ est vraie au rang $n+1$; or

et

$$
d_{n+1}=g_{n+1}\left(u_{n+1}\right)=g_{n}\left(u_{n+1}\right)+\chi_{b}^{I}(1 / 2)
$$

$$
g_{n}(x)=b^{2}\left(b^{2 n}-1\right)\left(x^{2}-u_{n}^{2}\right) / 12+\lambda_{n}\left(x-u_{n}\right)+g_{n}\left(u_{n}\right),
$$

d'où $d_{n+1}=d_{n}+\chi_{b}^{I}(1 / 2)+b^{2}\left(b^{2 n}-1\right)\left(u_{n+1}^{2}-u_{n}^{2}\right) / 12+\lambda_{n}\left(u_{n+1}-u_{n}\right)$, qui, après calcul, donne bien la formule souhaitée.

7.4. Démonstration du théorème 4.14. D'après la propriété 3.5(i), les pentes des segments de droite de la fonctions $\varphi_{b}^{\sigma}$ prennent une fois et une seule les valeurs $-b(b-1) / 2+j b$ pour $j=0,1, \ldots, b-1$; donc pour obtenir une permutation minimisant $t_{1}\left(S_{b}^{\sigma}\right)$, c'est-à-dire minimisant $\left|\varphi_{b}^{\sigma}\right|$, il est naturel de prendre les pentes des segments dans l'ordre suivant : $m,-(m+b),(m+2 b),-(m+3 b), \ldots$ où $m$ est la plus petite pente strictement positive.

7.4.1. Cas $b$ pair. Ici l'ordre des pentes de la fonction $\varphi=\varphi_{b}^{\sigma_{0}}$ est

$$
b / 2,-3 b / 2,5 b / 2, \ldots,-5 b / 2,3 b / 2,-b / 2,
$$

ce qui fournit la permutation $\sigma_{0}$ de l'énoncé par la formule

$$
\sigma_{0}(k-1)=(b-1) / 2-m_{k} / b
$$

où $m_{k}$ est la pente de $\varphi$ sur $[(k-1) / b, k / b]$. 
En appliquant la propriété 3.5(i), on obtient

$$
\varphi(k / b)=\varphi((b-k) / b)=(-1)^{k-1} k / 2 \quad \text { pour } 0 \leq k \leq b / 2,
$$

grâce à la symétrie de la fonction $\varphi$ par rapport à la droite $x=1 / 2$; il en résulte que le milieu de chaque segment du graphe de $\varphi$ a pour ordonnée $1 / 4$ si sa pente est positive et $-1 / 4$ sinon; il en résulte aussi que les points anguleux du graphe de $\varphi$ sont alternativement sur les droites $y=(b / 2) x$ et $y=-(b / 2) x$ sur $[0,1 / 2]$.

La fonction $g_{2}(x)=\varphi(x)+\varphi(x b)$ possède les propriétés :

$$
\max \left|g_{2}\right|=(b+1) / 4=\left|g_{2}(k / b+1 /(2 b))\right|,
$$

$k$ étant impair ou pair suivant que $b$ est multiple de 4 ou non; sur $[1 / 2-$ $\left.1 / b^{2}, 1 / 2\right]$, le segment du graphe de $g_{2}$ a pour pente $-(b-1) b / 2+(b / 2) b=b / 2$ ou $-b / 2$ suivant que $b$ est multiple de 4 ou non (cet intervalle est suggéré par le fait que $|\varphi|$ est dominée par sa restriction à $[1 / 2-1 /(2 b), 1 / 2]$ à translation et symétrie près).

D'une part,

$$
\beta_{b}^{\sigma_{0}}=\inf _{n \geq 1} d_{n} / n \leq d_{2} / 2=(b+1) / 8 \quad \text { puisque } \quad d_{2}=\max \left|g_{2}(x)\right| ;
$$

d'autre part, d'après le lemme 6.3.2, en prenant $a=\left(b^{2}-2\right) / 2$, on a

$$
2 \beta_{b}^{\sigma_{0}} \geq\left|g_{2}\left(a /\left(b^{2}-1\right)\right)\right|>|\varphi(1 / 2)|=b / 4
$$

car $1 / 2-1 / b^{2}<a /\left(b^{2}-1\right)<1 / 2$ et la pente de $g_{2}$ sur cet intervalle a le même signe que $\varphi(1 / 2)$.

D'où la conclusion du théorème dans le cas où $b$ est pair, d'après le théorème 4.9 .

7.4.2. Cas b impair. Ici l'ordre des pentes de $\varphi$ est

$$
b,-2 b, 3 b, \ldots,-3 b, 2 b,-b, 0,
$$

ce qui fournit la permutation $\sigma_{0}$ de l'énoncé. Remarquons que dans ce cas, la fonction $\varphi$ n'est plus symétrique par rapport à $x=1 / 2$, mais qu'elle l'est par rapport à $x=1 / 2-1 /(2 b)$, ce qui complique un peu les calculs bien que la méthode soit la même que dans le cas $b$ pair.

Nous commençons par les cas $b=1(\bmod 4)$.

Toujours d'après 3.5(i),

$$
\varphi(k / b)=(-1)^{k+1}[(k+1) / 2] \quad \text { pour } 0 \leq k \leq(b-1) / 2 ;
$$

il en résulte que le milieu de chaque segment du graphe de $\varphi$ a pour ordonnée $1 / 2$ si sa pente est positive et 0 sinon.

La fonction $g_{2}$ vérifie $\max \left|g_{2}\right|=\left(b^{2}+4 b-9\right) /(4 b)$; en effet, $|\varphi|$ est maximum en $1 / 2+1 /(2 b)$ et elle est dominée par sa restriction à $[1 / 2+$ $1 /(2 b), 1 / 2+1 / b]$ à translation et symétrie près, donc $\left|g_{2}\right|$ est maximum sur 
cet intervalle; or, pour $0 \leq k \leq(b-3) / 2$,

$$
\left|g_{2}\left(1 / 2+1 /(2 b)+k / b^{2}\right)\right| \leq\left|g_{2}\left(1 / 2+1 /(2 b)+(b-3) /\left(2 b^{2}\right)\right)\right|
$$

et le second membre vaut $(b-1) / 4+(5 b-9) /(4 b)$, d'où

$$
2 \beta_{b}^{\sigma_{0}} \leq d_{2}<(b+4) / 4 .
$$

Soit $a=b(b+1) / 2$; on a

$$
1 / 2+1 /(2 b)<a /\left(b^{2}-1\right)<1 / 2+1 /(2 b)+1 / b^{2}
$$

et la pente de $g_{2}$ sur cet intervalle vaut $-((b-3) / 2) b+b^{2}=b(b+3) / 2$; d'où

$$
\begin{aligned}
g_{2}\left(a /\left(b^{2}-1\right)\right) & =(b-1) / 4+(b(b+3) / 2)\left(a /\left(b^{2}-1\right)-(b+1) /(2 b)\right) \\
& =(b-1) / 4+(b+3) /(4(b-1)) \geq b / 4 .
\end{aligned}
$$

Finalement, grâce au lemme 6.3 .2 , on obtient $\beta_{b}^{\sigma_{0}} \geq b / 8$ et le théorème est démontré dans le cas $b=1(\bmod 4)$.

Traitons à présent le cas $b=3(\bmod 4)$.

La fonction $\varphi$ est dominée par sa restriction à $[1 / 2-1 /(2 b), 1 / 2]$ et, sur cet intervalle, sa pente vaut $-b(b-1) / 2$. On montre que, pour $0 \leq k \leq$ $(b-1) / 2$,

$$
\left|g_{2}\left(1 / 2-1 /(2 b)+k / b^{2}\right)\right| \leq\left|g_{2}\left(1 / 2-1 /(2 b)+(b-1) /\left(2 b^{2}\right)\right)\right|,
$$

ce qui donne $\beta_{b}^{\sigma_{0}} \leq(b+4) / 8$.

Soit $a=(b-1) b / 2$; on vérifie que

$$
1 / 2-1 /(2 b)<a /\left(b^{2}-1\right)<1 / 2-1 /(2 b)+1 / b^{2}
$$

et que, sur cet intervalle, la pente de $g_{2}$ vaut $b(b+1) / 2$; on en déduit

$$
\left|g_{2}\left(a /\left(b^{2}-1\right)\right)\right|=(b+2) / 4, \quad \text { d'où } \quad(b+2) / 8 \leq \beta_{b}^{\sigma_{0}} \leq(b+4) / 8,
$$

ce qui donne le théorème quand $b=3(\bmod 4)$.

\subsection{Démonstration des théorèmes $4.15,4.16$ et 4.17}

7.5.1. Démonstration du théorème 4.15. Pour le théorème 4.15 , le problème était dans un premier temps de trouver, pour une base donnée, les permutations dont le maximum des fonctions $\chi$ soit le plus faible possible. A cet effet, nous avons parcouru toutes les permutations par un programme récursif (langage Pascal) pour tester les fonctions $\chi$ associées; en fait, d'après le théorème 4.4 , nous avons pu nous borner aux permutations telles que $\sigma(0)=0$, ce qui a permis de limiter le temps de calcul. Dans ces conditions, nous avons obtenu les résultats suivants quant au nombre de permutations donnant le même plus petit maximum pour la fonction $\chi$ :

\begin{tabular}{ccccccccccc}
\hline base $b$ & 2 & 3 & 4 & 5 & 6 & 7 & 8 & 9 & 10 & 11 \\
\hline $\begin{array}{c}\text { nombre } \\
\text { de permutations }\end{array}$ & 1 & 2 & 2 & 8 & 12 & 8 & 32 & 32 & 8 & 1440 \\
\hline
\end{tabular}


Jusqu'à la base 10, toutes les permutations ainsi trouvées ont la même fonction $\chi$.

Par contre, n'en étant pas de même pour la base 11, nous avons effectué un tri supplémentaire en calculant le maximum de $g_{2}(x)=\chi(x)+$ $\chi(x b)$; après ce tri, il reste 128 permutations et, en comparant les résultats numériques obtenus pour les autres bases, nous n'avons pas jugé utile de pousser le tri plus loin.

Pour $b=2$ et 3 , la permutation identique faisant partie des permutations trouvées, la diaphonie est déjà calculée (voir théorème 4.13). Pour $b=4$, on constate que pour les permutations obtenues $\chi_{4}^{I}=4\left(\chi_{2}^{I}(x)+\chi_{2}^{I}(2 x)\right)$, d'où on déduit que $\gamma_{4}^{\sigma}=8 \gamma_{2}^{I}$ et, par suite, $f\left(S_{4}^{\sigma}\right)=f\left(S_{2}^{I}\right)$. Pour ces 3 bases, nous avons donc la valeur exacte de $f\left(S_{b}^{\sigma}\right)$.

Pour $5 \leq b \leq 11$, nous nous sommes bornés à des encadrements de $f\left(S_{b}^{\sigma}\right)$, notre but étant la recherche d'une base et d'une permutation donnant la plus petite valeur possible pour $f\left(S_{b}^{\sigma}\right)$. Les majorants sont obtenus en calculant le maximum de $g_{n}(x)$ (défini au lemme 6.3.2) pour des valeurs de $n$ comprises entre 3 et 6 , puis en appliquant le lemme 6.3.1. Les minorants résultent de l'application du lemme 6.3.2; nous avons calculé à la machine $g_{\nu}\left(a /\left(b^{\nu}-1\right)\right)$ pour $1 \leq a<b^{\nu}$, avec $\nu=1,2,3,4$; dans tous les cas le plus grand minorant est atteint pour $\nu=1$ ou 2 , ce qui nous fait penser que les minorants annoncés dans le théorème sont les valeurs exactes de $f\left(S_{b}^{\sigma}\right)$.

Ces encadrements nous permettent de sélectionner 10 comme étant la meilleure base jusqu'à 11, au regard de la faible diaphonie. Nous ne recherchons pas la valeur exacte de $f\left(S_{10}^{\sigma}\right)$ car le résultat obtenu serait de toute façon plus faible que celui du théorème 4.16.

7.5.2. Démonstration du thé or ème 4.16. Comme indiqué dans la remarque 3 suivant le théorème 4.15 , après une étude numérique analogue à celle qui précède pour les bases impaires entre 13 et 21 , nous examinons en détail le cas $b=19$ du théorème 4.16; notons auparavant que l'étude numérique donne, dans ce cas,

$$
1.315<f\left(S_{19}^{\sigma}\right)<1.452 .
$$

Expression de $\chi_{19}^{\sigma}$ ( $\sigma$ étant la permutation de théorème 4.16) : Il est facile de programmer le calcul de $\chi_{19}^{\sigma}$ en utilisant sa définition avec les fonctions $\varphi_{19, h}^{\sigma}$. Nous vérifions ainsi que le coefficient dominant vaut $10830=$ $19^{2}\left(19^{2}-1\right) / 12$ et que $\chi_{19}^{\sigma}$ est symétrique par rapport à $x=1 / 2$; il suffit donc de donner ses valeurs en $k / 19$ pour $0 \leq k \leq 9$ :

\begin{tabular}{ccccccccccc}
\hline$k$ & 0 & 1 & 2 & 3 & 4 & 5 & 6 & 7 & 8 & 9 \\
\hline$\chi_{19}^{\sigma}(k / 19)$ & 0 & 30 & 32 & 34 & 34 & 42 & 40 & 42 & 42 & 30 \\
\hline
\end{tabular}

D'après ce qui précède, on constate que $g_{1}=\chi$ est dominée par sa 
restriction à $\left[\frac{7}{19}, \frac{7.5}{19}\right]$ à symétrie et translation près. Pour $g_{2}$, le calcul sur machine montre qu'il y a deux intervalles dominants :

$$
\left[\frac{7}{19}+\frac{5}{19^{2}}, \frac{7}{19}+\frac{5.5}{19^{2}}\right] \quad \text { et }\left[\frac{7}{19}+\frac{7}{19^{2}}, \frac{7}{19}+\frac{7.5}{19^{2}}\right] .
$$

Par contre, pour $g_{3}$ on trouve à nouveau un seul intervalle dominant :

$$
\left[\frac{7}{19}+\frac{5}{19^{2}}+\frac{5}{19^{3}}, \frac{7}{19}+\frac{5}{19^{2}}+\frac{5.5}{19^{3}}\right] \text {. }
$$

Les remarques ci-dessus, ainsi que l'obtention du même minorant pour $1 \leq \nu \leq 4$ dans l'application du lemme 6.3.2, nous conduisent à formuler l'hypothèse de récurrence suivante, pour $n \geq 3$ :

La fonction $g_{n}$ admet un seul intervalle dominant

$$
\left[u_{n}, u_{n}+\frac{0.5}{19^{n}}\right] \quad \text { où } u_{n}=\frac{2}{19}+\frac{5}{18}\left(1-\frac{1}{19^{n}}\right) ;
$$

la fonction $g_{n}$ atteint son maximum $d_{n}$ en $u_{n}$ et, sur $\left[u_{n}, u_{n}+\frac{0.5}{19^{n}}\right]$, on a

$$
\begin{aligned}
g_{n}(x)= & \frac{19^{2}}{12}\left(19^{2 n}-1\right)\left(x-u_{n}\right)^{2} \\
& -\frac{1}{18^{2}}\left(10659 \times 19^{n}-17556-\frac{15}{19^{n-2}}\right)\left(x-u_{n}\right)+g_{n}\left(u_{n}\right)
\end{aligned}
$$

avec $g_{n}\left(u_{n}\right)=\frac{1913}{54} n+o(n)$.

Pour $n=3$ : Le calcul numérique montre que l'hypothèse est vraie et que

$$
g_{3}\left(u_{3}\right)=\chi\left(\frac{7}{19}+\frac{5}{19^{2}}+\frac{5}{19^{3}}\right)+\chi\left(\frac{5}{19}+\frac{5}{19^{2}}\right)+\chi\left(\frac{5}{19}\right)=113.648 \ldots
$$

Passage du rang $n$ au rang $n+1$ : Pour $0 \leq k \leq 9$, posons

$$
y_{k}=g_{n+1}\left(u_{n}+\frac{k}{19^{n+1}}\right)=g_{n}\left(u_{n}+\frac{k}{19^{n+1}}\right)+\chi\left(\frac{k}{19}\right) .
$$

Commençons tout d'abord par comparer les valeurs $y_{k}$. On a

$y_{k+1}-y_{k}=\left(1-\frac{1}{19^{2 n}}\right) \frac{k}{6}+\frac{1}{18^{2}}\left(-534+\frac{924}{19^{n}}+\frac{258}{19^{2 n}}\right)+\chi\left(\frac{k+1}{19}\right)-\chi\left(\frac{k}{19}\right)$,

d'où on déduit que

$$
y_{0}<y_{10}<y_{9}<y_{1}<y_{4}<y_{2}<y_{3}<y_{6}<y_{8}<y_{7}<y_{5} .
$$

Sur ces inégalités, on voit que les seuls intervalles dominants possibles pour $g_{n+1}$ sont

$$
\left[u_{n}+\frac{5}{19^{n+1}}, u_{n}+\frac{5.5}{19^{n+1}}\right] \text { et } \quad\left[u_{n}+\frac{7}{19^{n+1}}, u_{n}+\frac{7.5}{19^{n+1}}\right] .
$$


Pour les départager, nous calculons

$$
g_{n+1}\left(u_{n}+\frac{5.5}{19^{n+1}}\right)-g_{n+1}\left(u_{n}+\frac{7.5}{19^{n+1}}\right)=\frac{1}{18^{2}}\left(96-\frac{1848}{19^{n}}+\frac{132}{19^{2 n}}\right),
$$

quantité positive dès que $n \geq 2$.

En conséquence, nous avons démontré que $g_{n+1}$ admet $\left[u_{n+1}, u_{n+1}+\right.$ $\left.\frac{0.5}{19^{n+1}}\right]$ pour seul intervalle dominant, puisque $u_{n+1}=u_{n}+\frac{5}{19^{n+1}}$ et que $d_{n+1}=\max g_{n+1}=g_{n+1}\left(u_{n+1}\right)$.

Reste à calculer $g_{n+1}(x) \operatorname{sur}\left[u_{n+1}, u_{n+1}+\frac{0.5}{19^{n+1}}\right]$ :

$$
g_{n+1}(x)=g_{n}(x)+\chi\left(19^{n} x\right)=g_{n}(x)+\chi\left(19^{n}\left(x-u_{n}\right)\right) ;
$$

or, $\operatorname{sur}\left[\frac{5}{19}, \frac{5.5}{19}\right], \chi(z)=10830 z^{2}-6308 z+952$, d'où

$$
\begin{aligned}
& g_{n+1}(x)=\left(\frac{19^{2}}{12}\left(19^{2 n}-1\right)+10830 \times 19^{2 n}\right)\left(x-u_{n}\right)^{2} \\
& +\left(\frac{1}{18^{2}}\left(-10659 \times 19^{n}+17556+\frac{15}{19^{n-2}}\right)-19^{n} \times 6308\right)\left(x-u_{n}\right) \\
& +g_{n}\left(u_{n}\right)+952,
\end{aligned}
$$

c'est-à-dire, en utilisant la relation $u_{n+1}=u_{n}+\frac{5}{19^{n+1}}$,

$$
\begin{aligned}
g_{n+1}(x)= & \frac{19^{2}}{12}\left(19^{2 n+2}-1\right)\left(x-u_{n+1}\right)^{2} \\
& -\frac{1}{18^{2}}\left(10659 \times 19^{n+1}-17556-\frac{15}{19^{n-1}}\right)\left(x-u_{n+1}\right) \\
& +g_{n}\left(u_{n}\right)+\frac{1913}{54}+\frac{4620}{18^{2} \times 19^{n}}+\frac{750}{18^{2} \times 19^{2 n}},
\end{aligned}
$$

ce qui donne

$$
g_{n+1}\left(u_{n+1}\right)=g_{n}\left(u_{n}\right)+\frac{1913}{54}+o(1) .
$$

Finalement, l'hypothèse de récurrence est bien vérifiée au rang $n+1$; ce qui achève la démonstration du théorème 4.16 , d'après le théorème 4.10 .

Remarquons que le théorème 4.17 est conséquence immédiate des théorèmes $4.11,4.15$ et 4.16 .

7.6. Démonstration des théorèmes 4.18 et 4.19. Rappelons que, pour $B_{n-1} \leq N<B_{n}$, on a

$$
D\left(N, S_{B}^{\Sigma}\right)=\sum_{j=1}^{n} \psi_{b_{j}}^{\sigma_{j-1}}\left(N / B_{j}\right)+\sum_{j=n+1}^{\infty} \psi_{b_{j}}^{\sigma_{j-1}}\left(N / B_{j}\right)
$$

et que la deuxième sommation est inférieure à 1 (voir 6.1). 
D'après la construction des suites $\Sigma_{0}$, on a

$$
\psi_{b_{j}}^{\sigma_{j-1}} \leq d_{b_{j}}^{\sigma_{j-1}} \leq \log b_{j} / \log 2,
$$

d'où

$$
D\left(N, S_{B}^{\Sigma_{0}}\right) \leq 1+\left(\log N+\log b_{n}\right) / \log 2 .
$$

Si $B$ vérifie la deuxième hypothèse du théorème 4.18 , on obtient

$$
D\left(N, S_{B}^{\Sigma_{0}}\right) \leq 1+(K+1) \log N / \log 2 .
$$

Remarquons que, d'après le théorème 4.5 , la première hypothèse du théorème 4.18 n'est pas nécessaire pour avoir le résultat annoncé.

Rappelons que

$$
\begin{aligned}
F^{2}\left(N, S_{B}^{\Sigma}\right) & =4 \pi^{2} \sum_{j=1}^{\infty} \chi_{b_{j}}^{\sigma_{j-1}}\left(N / B_{j}\right) / b_{j}^{2} \\
& =4 \pi^{2} \sum_{j=1}^{\infty} \sum_{0 \leq h<k \leq b_{j}-1}\left(\varphi_{h, b_{j}}^{\sigma_{j-1}}-\varphi_{k, b_{j}}^{\sigma_{j-1}}\right)^{2}\left(N / B_{j}\right) / b_{j}^{2},
\end{aligned}
$$

et que, si $B_{n-1} \leq N<B_{n}$,

$$
\sum_{j=n+1}^{\infty} \chi_{b_{j}}^{\sigma_{j-1}}\left(N / B_{j}\right) / b_{j}^{2}<1 / 12 \quad(\text { voir } 6.1) .
$$

Par ailleurs $\left|\varphi_{h, b_{j}}^{\sigma_{j-1}}-\varphi_{k, b_{j}}^{\sigma_{j-1}}\right| \leq \psi_{b_{j}}^{\sigma_{j-1}}$, d'où

$$
F^{2}\left(N, S_{B}^{\Sigma_{0}}\right) \leq 4 \pi^{2}\left(1 / 12+\sum_{j=1}^{n}\left(b_{j}-1\right)\left(\log b_{j}\right)^{2} /\left(2 b_{j}(\log 2)^{2}\right)\right),
$$

ce qui fournit

$$
F^{2}\left(N, S_{B}^{\Sigma_{0}}\right)<\pi^{2} / 3+2(\pi / \log 2)^{2} \sum_{j=1}^{n}\left(\log b_{j}\right)^{2} .
$$

Si $B$ vérifie la deuxième hypothèse du théorème 4.19 , on obtient

$$
F^{2}\left(N, S_{B}^{\Sigma_{0}}\right) \leq \pi^{2} / 3+2 \pi^{2} K(\log N / \log b+1) /(\log 2)^{2} .
$$

Ici aussi, la première hypothèse du théorème 4.19 assure qu'on n'est pas dans le cas du théorème 4.6.

\section{Références}

[1] E. Y. Atanassov, Note on the discrepancy of the van der Corput generalized sequences, C. R. Acad. Bulgare Sci. 42 (3) (1989), 41-44.

[2] E. Braaten and G. Weller, An improved low discrepancy sequence for multidimensional quasi-Monte-Carlo integration, J. Comput. Phys. 33 (1979), 249-258. 
[3] H. Chaix et H. Faure, Discrépance et diaphonie des suites de van der Corput généralisées, C. R. Acad. Sci. Paris Sér. I Math. 310 (1990), 315-320.

[4] -, -, Discrépance et diaphonie des suites de van der Corput généralisées (II), ibid. 311 (1990), 65-68.

[5] - - - Discrépance et diaphonie des suites de van der Corput généralisées (III), ibid. 312 (1991), 755-758.

[6] H. Faure, Discrépance de suites associées à un système de numération (en dimension un), Bull. Soc. Math. France 109 (1981), 143-182.

[7] —, Discrépance quadratique de la suite de van der Corput et de sa symétrique, Acta Arith. 55 (1990), 333-350.

[8] —, Good permutations for extreme discrepancy, J. Number Theory, à paraître.

[9] L. Kuipers and H. Nied erreiter, Uniform Distribution of Sequences, Wiley, New York 1974

[10] G. Pagès, Van der Corput sequences, Kakutani transforms and one-dimensional numerical integration, prépublication du CERMA, Ecole Nationale des Ponts et Chaussées, 1990.

[11] P. D. Proinov, Estimation of $L^{2}$-discrepancy of a class of infinite sequences, C. R. Acad. Bulgare Sci. 36 (1) (1983), 37-40.

[12] -, On irregularities of distribution, ibid. 39 (9) (1986), 31-34.

[13] - Symmetrization of the van der Corput generalized sequences, Proc. Japan Acad. Ser. A Math. Sci. 64 (5) (1988), 159-162.

[14] P. D. Proinov and E. Y. Atanassov, On the distribution of the van der Corput generalized sequences, C. R. Acad. Sci. Paris Sér. I Math. 307 (1988), 895-900.

[15] P. D. Proinov and V. S. Grozdanov, Symmetrization of the van der CorputHalton sequence, C. R. Acad. Bulgare Sci. 40 (8) (1987), 5-8.

[16] - - - On the diaphony of the van der Corput-Halton sequence, J. Number Theory 30 (1988), 94-104.

[17] Y. J. Xiao, Suites équiréparties associées aux automorphismes du tore, C. R. Acad. Sci. Paris Sér. I Math. 311 (1990), 579-582.

[18] —, Contributions aux méthodes arithmétiques pour la simulation accélérée, Thèse, Ecole Nationale des Ponts et Chaussées, Septembre 1990.

[19] P. Zinterhof, Über einige Abschätzungen bei der Approximation von Funktionen mit Gleichverteilungsmethoden, Österreich. Akad. Wiss. Math.-Natur. Sitzungsber. II 185 (1976), 121-132.

[20] P. Zinterhof und H. Stegbuchner, Trigonometrische Approximation mit Gleichverteilungsmethoden, Studia Sci. Math. Hungar. 13 (1978), 273-289.

UFR-MIM et URA 225

UNIVERSITÉ DE PROVENCE

3 PLACE VICTOR HUGO

13331 MARSEILLE CEDEX 3, FRANCE

Reçu le 2.12.1991

et révisé le 14.6.1992 